\title{
Maximum Palinstrophy Growth in 2D Incompressible Flows
}

\author{
Diego Ayala and Bartosz Protas* \\ Department of Mathematics and Statistics, McMaster University \\ Hamilton, Ontario, L8S 4K1, Canada
}

December 19, 2013

\begin{abstract}
In this study we investigate vortex structures which lead to the maximum possible growth of palinstrophy in two-dimensional incompressible flows on a periodic domain. The issue of palinstrophy growth is related to a broader research program focusing on extreme amplification of vorticity-related quantities which may signal singularity formation in different flow models. Such extreme vortex flows are found systematically via numerical solution of suitable variational optimization problems. We identify several families of maximizing solutions parameterized by their palinstrophy, palinstrophy and energy and palinstrophy and enstrophy. Evidence is shown that some of these families saturate estimates for the instantaneous rate of growth of palinstrophy obtained using rigorous methods of mathematical analysis, thereby demonstrating that this analysis is in fact sharp. In the limit of small palinstrophies the optimal vortex structures are found analytically, whereas for large palinstrophies they exhibit a self-similar multipolar structure. It is also shown that the time evolution obtained using the instantaneously optimal states with fixed energy and palinstrophy as the initial data saturates the upper bound for the maximum growth of palinstrophy in finite time. Possible implications of this finding for the questions concerning extreme behavior of flows are discussed.
\end{abstract}

Keywords: 2D Navier-Stokes equation, maximum growth, palinstrophy, variational optimization, vortex dynamics

\section{Introduction}

This work makes a contribution to a broader research effort concerning systematic characterization of extreme events in hydrodynamic systems. In addition to their independent physical interest, such questions are intrinsically related to the problem of finitetime singularity formation in various flow models, which is one of the issues at the

*Email address for correspondence: bprotas@mcmaster.ca 
center of mathematical fluid mechanics. In the context of the three-dimensional (3D) Navier-Stokes system in an unbounded or periodic domain, the key problem concerns the existence for arbitrarily large times of smooth solutions corresponding to arbitrary initial data (Doering, 2009). To date, global in time existence has been established for weak solutions only, which need not be smooth. On the other hand, for initial data of arbitrary size, smooth (classical) solutions are guaranteed to exist up to certain finite times only, and loss of regularity, referred to as "blow-up", cannot be ruled out. The importance of this issue has been recognized by the Clay Mathematical Institute which identified it as one of the "millennium challenges" for the mathematics community with a suitable monetary prize (Fefferman, 2000). Similar questions concerning existence of smooth solutions also pertain to the Euler equations in 3D. While the problem is essentially one of mathematical analysis, a number of computational investigations have been undertaken (e.g., Brachet et al., 1983; Brachet, 1991; Kerr, 1993; Hou, 2009; Pelz, 2001; Ohkitani \& Constantin, 2008; Ohkitani, 2008; Grafke et al., 2008; Gibbon et al., 2008; Orlandi et al., 2012), to understand whether or not blow-up may occur in finite time. Although some of these studies indicated the possibility of a blow-up, the results obtained to date are not conclusive and their interpretation remains the subject of a debate. Another related research direction involves the study of complex-valued extensions of the Euler and Navier-Stokes equations. The idea is that solutions to the equations which at some fixed time are real-analytic functions of the space variables possess singularities in the complex plane, and the distance from the real axis to the nearest singularity, referred to as the width of the analyticity strip (Sulem et al., 1983), further characterizes the smoothness of the solution. Therefore, migration of such complex-plane singularities towards the real line might be a signature of an approaching blow-up. In the context of this approach we only mention recent studies by Matsumoto et al. (2008); Siegel \& Caflisch (2009); Bustamante \& Brachet (2012), and refer the reader to the references quoted therein for further details. A common limitation of these earlier attempts is that the candidates for blow-up (given in terms of the initial data for the Navier-Stokes or Euler equations) were chosen in a rather ad-hoc manner based on some heuristic arguments. A long-term goal of the present research program is to conduct the search for potential finite-time singularities in hydrodynamic systems more systematically, leveraging modern methods of numerical optimization.

In mathematical analysis there are many different lines of attack on the Navier-Stokes regularity problem. One important approach relies on estimates for the growth of the enstrophy $\mathcal{E}(t):=\frac{1}{2} \int_{\Omega}|\boldsymbol{\nabla} \times \mathbf{u}(t, \mathbf{x})|^{2} d \Omega$, where $\mathbf{u}(t, \cdot): \Omega \rightarrow \mathbb{R}^{3}$ is the velocity field and $\Omega$ is a $3 \mathrm{D}$ domain (periodic or unbounded). It is well known (Foias \& Temam, 1989) that the loss of regularity will manifest itself by the enstrophy becoming unbounded $\mathcal{E}(t) \rightarrow \infty$ as $t \rightarrow t_{0}$, where $t_{0}$ is the blow-up time. Therefore, it is essential to provide tight bounds on how rapidly the enstrophy can grow, and the sharpest estimate available to date for the 3D Navier-Stokes system has the form (Doering \& Gibbon, 1995; Doering, 2009)

$$
\frac{d \mathcal{E}(t)}{d t} \leq \frac{27 C^{3}}{32 \nu^{3}} \mathcal{E}(t)^{3}
$$


where $\nu$ is the kinematic viscosity and $C>0$ is a constant (hereafter $C$ will denote a generic positive constant which may assume different numerical values in different instances). Results similar to (1) were developed earlier by Serrin (1963) and Ladyzhenskaya (1969). Since upon integration with respect to time this upper bound blows up at $t_{0}=\frac{16 \nu^{3}}{27 C^{3} \mathcal{E}_{0}^{2}}$, where $\mathcal{E}_{0}$ is the initial value of the enstrophy, the regularity problem can be rephrased as the question whether or not estimate (1) can be saturated uniformly during the system evolution over a finite window of time $[0, T]$, where $T<t_{0}$. In other words, the question is whether there exists initial data $\mathbf{u}_{0}$ with some prescribed enstrophy $\mathcal{E}_{0}$ such that the corresponding system evolution will realize estimate (1) over a finite time window $[0, T]$. Such initial data can be sought via solution of a suitably formulated variational optimization problem for partial differential equation (PDEs) in which the objective is to maximize the growth of the enstrophy.

In order to investigate the possibility of a finite-time blow-up, two questions need to be addressed, namely:

(P1) Sharpness of instantaneous estimate (1), and

(P2) the maximum growth of enstrophy over finite time window $[0, T]$, which is mathematically defined as

$$
\max _{\mathbf{u}_{0} \in H^{1}(\Omega), \mathcal{E}(0)=\mathcal{E}_{0}} \mathcal{E}(T)
$$

By solving optimization problem (2) over a set of time windows with increasing length $T$ one could assess whether or not the worst-case growth of enstrophy indeed exhibits a tendency towards blow-up in finite time. Moreover, this will also shed light on the structure of the most singular initial data which can lead to new conjectures in the mathematical analysis of the problem. In the context of the 3D Navier-Stokes system problem P1 was already addressed in the seminal study by Lu \& Doering (2008) (see also Lu, 2006), where it was demonstrated using computations that estimate (1) is in fact sharp (up to a prefactor). From the computational point of view, solution of problems P1 and P2 is based on a form of the discrete gradient flow. Needless to say, this approach is much more complicated in the case of open problem P2, since in order to compute the gradient directions, the time-dependent Navier-Stokes system and its suitably defined adjoint have to be solved. While this is a formidable computational task, it does appear within reach of the computational techniques and resources available to date. At this point it should be made clear that, although solving problem P2 is the long-term objective of the present research program, accomplishing this task will not resolve the Clay Millennium Problem where a rigorous mathematical proof is required (Fefferman, 2000).

In analogy with problems P1 and P2, questions concerning the maximum possible growth of various quantities can also be formulated in regard to the two-dimensional (2D) Navier-Stokes and one-dimensional (1D) Burgers equations. While for both of these systems it is well known that smooth solutions exist globally in time for arbitrary smooth initial data (Kreiss \& Lorenz, 2004), one can also obtain estimates for both the 
instantaneous and finite-time growth of the relevant quadratic quantities and it is important to know whether these estimates are sharp and can be attained during the nonlinear evolution of the system. Our interest is justified by the fact that these estimates are obtained using similar techniques as employed in the analysis of the 3D Navier-Stokes problem. The quantities of interest are the "enstrophy" $\mathcal{E}(t):=\frac{1}{2} \int_{0}^{1}\left(\partial_{x} v(t, x)\right)^{2} d x$ in $1 \mathrm{D}$, where $v: \mathbb{R}^{+} \times[0,1] \rightarrow \mathbb{R}$ is the solution of the Burgers equation, and the palinstrophy $\mathcal{P}(t):=\frac{1}{2} \int_{\Omega}|\boldsymbol{\nabla} \omega(t, \mathbf{x})|^{2} d \Omega$ in $2 \mathrm{D}$, where $\Omega:=[0,1] \times[0,1]$ and $\omega: \mathbb{R}^{+} \times \Omega \rightarrow \mathbb{R}$ is the scalar vorticity (as will be discussed further below, enstrophy is not interesting in $2 \mathrm{D}$, since in the absence of any right-hand side forcing, it may not increase in flows on periodic and unbounded domains).

In this investigation we are interested in assessing the sharpness of certain analytic estimates for the instantaneous and finite-time growth of palinstrophy with respect to variations of the palinstrophy in the presence of suitable side constraints. In other words, we will seek upper bounds which are slowest-growing functions of the palinstrophy with some other quantity fixed. An estimate is thus declared "sharp" if there exists a family of fields unconstrained by flow evolution which exhibits the same growth of $d \mathcal{P} / d t$ or $\max _{t \geq 0} \mathcal{P}(t)$ with increasing palinstrophy as predicted by the estimate (up to a constant prefactor). Since such notion of estimate sharpness does not explicitly involve flow evolution (which is considered only a posteriori), an estimate may be sharp even if it does not have an optimal structure with respect to the time evolution (this point is further discussed in section 2 below). We also remark that, in principle, depending on the structure of the side constraints, estimates exhibiting different power-law dependence on the palinstrophy may simultaneously be sharp. In addition to being physically relevant, such formulation leads to precise and computationally verifiable criteria for sharpness.

Our focus will be on upper bounds expressed in terms of quadratic quantities, namely, energy $\mathcal{K}$, enstrophy $\mathcal{E}$ and palinstrophy $\mathcal{P}$. Selected estimates for problems analogous to problems P1 and P2 formulated for the 1D Burgers and 2D Navier-Stokes systems, together with the aforementioned results for the 3D Navier-Stokes system, are summarized in table 1. Determining whether or not the estimates listed in table 1 are sharp in the sense made precise above and, if so, identifying the solutions which saturate these estimates constitutes the long-term goal of this research program. In fact, significant progress has already been made addressing some of these questions. The instantaneous bound on $d \mathcal{E} / d t$ for the 1D Burgers problem was shown to be sharp by Lu \& Doering (2008) (see also Lu, 2006), and a remarkable feature of this result is that it was obtained analytically. Finite-time estimates for the 1D Burgers problem were probed computationally by Ayala \& Protas (2011), where it was shown that they are not in fact sharp. This result is important, as it suggests that the standard way for performing analysis based on integrating (sharp) instantaneous bounds over time might not be optimal and might lead to significant overestimates. The results obtained numerically by Ayala \& Protas (2011) were then justified rigorously by Pelinovsky $(2012 a, b)$. We emphasize that, based on the results in the $1 \mathrm{D}$ and $2 \mathrm{D}$ cases, it is not possible to speculate about the maximum growth of various quantities in $3 \mathrm{D}$ flows. We add here that variational optimization methods have recently been employed to study other fundamental problems 


\begin{tabular}{|c|c|c|}
\hline & Estimate & SHARPNESS \\
\hline $\begin{array}{l}\text { 1D Burgers } \\
\text { instantaneous }\end{array}$ & $\frac{d \mathcal{E}}{d t} \leq \frac{3}{2}\left(\frac{1}{\pi^{2} \nu}\right)^{1 / 3} \mathcal{E}^{5 / 3}$ & $\begin{array}{c}\text { YES } \\
\text { (Lu \& Doering, 2008) }\end{array}$ \\
\hline $\begin{array}{l}\text { 1D Burgers } \\
\text { finite-time }\end{array}$ & $\max _{t \in[0, T]} \mathcal{E}(t) \leq\left[\mathcal{E}_{0}^{1 / 3}+\frac{1}{16}\left(\frac{1}{\pi^{2} \nu}\right)^{4 / 3} \mathcal{E}_{0}\right]^{3}$ & $\begin{array}{c}\text { No } \\
\text { (Ayala \& Protas, } \\
2011)\end{array}$ \\
\hline $\begin{array}{l}\text { 2D Navier-Stokes } \\
\text { instantaneous }\end{array}$ & $\begin{array}{c}\frac{d \mathcal{P}(t)}{d t} \leq-\nu \frac{\mathcal{P}^{2}}{\mathcal{E}}+\frac{C_{1}}{\nu} \mathcal{E} \mathcal{P} \\
\frac{d \mathcal{P}(t)}{d t} \leq \frac{C_{2}}{\nu} \mathcal{K}^{1 / 2} \mathcal{P}^{3 / 2}\end{array}$ & present work \\
\hline $\begin{array}{l}\text { 2D Navier-Stokes } \\
\text { finite-time }\end{array}$ & $\begin{array}{c}\max _{t>0} \mathcal{P}(t) \leq \mathcal{P}_{0}+\frac{C_{1}}{2 \nu^{2}} \mathcal{E}_{0}^{2} \\
\max _{t>0} \mathcal{P}(t) \leq\left(\mathcal{P}_{0}^{1 / 2}+\frac{C_{2}}{4 \nu^{2}} \mathcal{K}_{0}^{1 / 2} \mathcal{E}_{0}\right)^{2}\end{array}$ & present work \\
\hline $\begin{array}{l}\text { 3D Navier-Stokes } \\
\text { instantaneous }\end{array}$ & $\frac{d \mathcal{E}(t)}{d t} \leq \frac{27 C^{2}}{32 \nu^{3}} \mathcal{E}(t)^{3}$ & $\begin{array}{l}\text { YES } \\
(\mathrm{Lu} \& \text { Doering, } \\
2008)\end{array}$ \\
\hline $\begin{array}{l}\text { 3D Navier-Stokes } \\
\text { finite-time }\end{array}$ & $\mathcal{E}(t) \leq \frac{\mathcal{E}(0)}{\sqrt{1-4 \frac{C \mathcal{E}(0)^{2}}{\nu^{3}} t}}$ & ??? \\
\hline
\end{tabular}

Table 1: Summary of selected estimates for the instantaneous rate of growth and the growth over finite time of enstrophy and palinstrophy in 1D Burgers, 2D and 3D NavierStokes systems.

in hydrodynamics such as the realizability of the Kraichnan-Leith-Batchelor theory of the 2D turbulence (Farazmand et al., 2011) and also involving the growth of quadratic quantities, e.g., optimal perturbations in the laminar-turbulent transition (Rabin et al., 2012).

In this study we report new results concerning the realizability of analytic bounds for $d \mathcal{P} / d t$ and $\max _{t>0} \mathcal{P}(t)$ in the 2D Navier-Stokes problem. It should be noted that, given the structure of the corresponding extreme vortex states, these results are also quite interesting from the physical point of view, outside the context of the sharpness of mathematical analysis. The structure of the paper is as follows: in the next section we discuss a number of rigorous estimates of the palinstrophy growth in the 2D Navier-Stokes system. In section 3 we demonstrate how questions about the sharpness of these estimates can be framed in terms of suitable variational optimization problems. A gradient-based approach to solution of such problems is discussed in section 4, whereas some analytical insights concerning the solutions of the maximization problems in the limit of small palinstrophies are presented in section 5. Computational results are presented in section 6 and discussed in section 7. Conclusions and a discussion of some future research directions are deferred to section 8. Some technical material is collected in an appendix. 


\section{Two-Dimensional Navier-Stokes System}

We consider a viscous incompressible fluid on a $2 \mathrm{D}$ periodic domain $\Omega=[0,1] \times[0,1]$. Its motion is governed by the Navier-Stokes equation, written here in the form

$$
\begin{array}{ll}
\frac{\partial \omega}{\partial t}+J(\omega, \psi)=\nu \Delta \omega & \text { in }(0, \infty) \times \Omega, \\
-\Delta \psi=\omega & \text { in }(0, \infty) \times \Omega, \\
\omega(0)=\omega_{0} & \text { in } \Omega,
\end{array}
$$

where $\psi$ and $\omega$ are, respectively, the streamfunction and (scalar) vorticity, whereas $\omega_{0}$ is the initial condition. In system (3) $\nu$ denotes the kinematic viscosity (assumed fixed), $\Delta$ is the Laplacian operator and $J(f, g):=\partial_{x} f \partial_{y} g-\partial_{y} f \partial_{x} g$, defined for $f, g: \Omega \rightarrow \mathbb{R}$, is the Jacobian determinant. Discussion concerning various aspects of formulation (3) can be found, for example, in Majda \& Bertozzi (2002).

We are interested in studying the growth of the following quadratic quantities characterizing the evolution of system (3)

$$
\begin{array}{ll}
\text { kinetic energy } & \mathcal{K}(\psi(t))=\frac{1}{2} \int_{\Omega}|\boldsymbol{\nabla} \psi(t, \mathbf{x})|^{2} d \Omega, \\
\text { enstrophy } & \mathcal{E}(\psi(t))=\frac{1}{2} \int_{\Omega}(\Delta \psi(t, \mathbf{x}))^{2} d \Omega, \\
\text { palinstrophy } & \mathcal{P}(\psi(t))=\frac{1}{2} \int_{\Omega}|\boldsymbol{\nabla} \Delta \psi(t, \mathbf{x})|^{2} d \Omega
\end{array}
$$

which, to simplify our analysis, are rewritten here in terms of streamfunction as the state variable. Without loss of generality, we will assume that the streamfunction fields have zero mean. As regards enstrophy (5), we note that multiplying (3a) by $\omega$, integrating the resulting expression over $\Omega$, performing integration by parts and making necessary simplifications, we arrive at

$$
\frac{d \mathcal{E}}{d t}=-2 \nu \mathcal{P} \leq 0
$$

which implies that, unlike in the dimension one or three, in 2D flows on periodic domains the enstrophy cannot increase (it will, in fact, decrease unless the vorticity is constant, or the fluid is inviscid, i.e., $\nu=0$ ). This result (which also holds on unbounded domains) is a consequence of the absence of the "vortex stretching" term in the $2 \mathrm{D}$ vorticity equation (3a). On the other hand, the phenomenon of stretching is observed (in the form of the last term on the right-hand side) in the evolution equation for the vorticity gradient $\nabla \omega$ which is obtained by applying the gradient operator $\boldsymbol{\nabla}$ to equation (3a)

$$
\frac{\partial \boldsymbol{\nabla} \omega}{\partial t}+(\mathbf{u} \cdot \boldsymbol{\nabla}) \boldsymbol{\nabla} \omega=\nu \Delta \boldsymbol{\nabla} \omega-[\boldsymbol{\nabla} \mathbf{u}]^{T} \cdot \boldsymbol{\nabla} \omega
$$

For clarity, this equation is written using the velocity field $\mathbf{u}=\left[\frac{\partial \psi}{\partial y},-\frac{\partial \psi}{\partial x}\right]^{T}$. Palinstrophy (6) is the quadratic quantity associated with equation (8), and a relation characterizing 
its evolution in time is obtained by dotting equation (8) with $\nabla \omega$, integrating over $\Omega$, then integrating by parts and simplifying

$$
\frac{d \mathcal{P}(t)}{d t}=\int_{\Omega} J(\Delta \psi, \psi) \Delta^{2} \psi d \Omega-\nu \int_{\Omega}\left(\Delta^{2} \psi\right)^{2} d \Omega=: \mathcal{R}_{\mathcal{P}}(\psi),
$$

where the subscript $\mathcal{P}$ indicates the value of the palinstrophy for which the expression is evaluated. We note that now, unlike in equation (7), the right-hand side (RHS) features a cubic term representing stretching in addition to the negative-definite dissipative term. We add that an equivalent expression for $d \mathcal{P} / d t$ was also obtained by Tran \& Dritschel (2006).

Since palinstrophy may exhibit nontrivial behavior, we now go on to discuss various rigorous bounds available for the palinstrophy rate of growth (9). The following estimate was recently obtained by Doering \& Lunasin (2011)

$$
\frac{d \mathcal{P}}{d t} \leq-\nu \frac{\mathcal{P}^{2}}{\mathcal{E}}+\frac{C_{1}}{\nu} \mathcal{E} \mathcal{P}
$$

A different estimate is derived in appendix $\mathrm{A}$ and has the form

$$
\frac{d \mathcal{P}}{d t} \leq \frac{C_{2}}{\nu} \mathcal{K}^{\frac{1}{2}} \mathcal{P}^{\frac{3}{2}}
$$

We observe that, in comparison to the corresponding estimates available in 1D and in 3D (see table 1), bounds (10) and (11) have a different structure, since the RHS expressions depend on two quadratic quantities, respectively, $\mathcal{E}$ and $\mathcal{P}$ in (10), and $\mathcal{K}$ and $\mathcal{P}$ in (11), rather than just one. In principle, the second quantity $(\mathcal{E}$ or $\mathcal{K})$ can be eliminated using Poincaré's inequality $\left[\mathcal{K} \leq(2 \pi)^{-2} \mathcal{E} \leq(2 \pi)^{-4} \mathcal{P}\right]$ yielding

$$
\frac{d \mathcal{P}}{d t} \leq \frac{C}{\nu} \mathcal{P}^{2}
$$

(transforming (10) into (12) also requires dropping the negative-definite quadratic term). We note however that, since the only functions saturating Poincarés inequality are the eigenfunctions of the Laplacian, such transformation of (10) and (11) into (12) may not be optimal, resulting in the possible loss of sharpness. Establishing whether or not upper bounds (10), (11) and (12) are sharp with respect to variations of palinstrophy $\mathcal{P}$, and determining the structure of the corresponding maximizing fields is the main goal of the present study.

As regards the maximum growth of palinstrophy over finite time, we notice that, although straightforward integration of (10) and (11) leads to unbounded increase of $\mathcal{P}$, when additional relations are used in the process, namely $d \mathcal{K} / d t=-2 \nu \mathcal{E}$ and $d \mathcal{E} / d t=$ $-2 \nu \mathcal{P}$ (cf. (7)), then bounded growth is in fact obtained in finite time. Starting from estimate (10), Doering \& Lunasin (2011) found that

$$
\max _{t>0} \mathcal{P}(t) \leq \mathcal{P}(0)+\frac{C_{1}}{2 \nu^{2}} \mathcal{E}(0)^{2}
$$


Similarly, it follows from estimate (11) that, cf. appendix A,

$$
\max _{t>0} \mathcal{P}(t) \leq\left(\mathcal{P}^{1 / 2}(0)+\frac{C_{2}}{4 \nu^{2}} \mathcal{K}^{1 / 2}(0) \mathcal{E}(0)\right)^{2} .
$$

It is worth noticing that, although finite-time estimates (13) and (14) are obtained from two different instantaneous estimates, they both give the same power-law behavior $\max _{t>0} \mathcal{P}(t) \sim \mathcal{P}(0)$ in the limit $\mathcal{P}(0) \rightarrow 0$. Similarly, the two estimates reduce to $\max _{t>0} \mathcal{P}(t) \sim \mathcal{E}(0)^{2}$ in the limit $\mathcal{E}(0) \rightarrow \infty$ (assuming that $\mathcal{K}(0)$ is fixed in (14)).

We add that some other estimates for the growth of palinstrophy were also derived in the literature. For example, the following bounds were established by Tran \& Dritschel (2006)

$$
\begin{aligned}
\frac{d \mathcal{P}}{d t} & \leq \frac{\|\Delta \omega\|_{L_{2}(\Omega)}}{\sqrt{2 \mathcal{E}}}\left(\|\omega\|_{L_{\infty}(\Omega)} \mathcal{E}-\nu \mathcal{P}\right), \\
\mathcal{P}(t) & \leq \frac{\left\|\omega_{0}\right\|_{L_{\infty}(\Omega)} \mathcal{E}(0)}{\nu}, \quad \text { for } t>0,
\end{aligned}
$$

whereas using a logarithmic bound on $\|\boldsymbol{\nabla} \mathbf{u}\|_{L_{\infty}(\Omega)}$, cf. Doering \& Gibbon (1995), it is possible to deduce

$$
\frac{d \mathcal{P}}{d t} \leq C\|\omega\|_{L_{\infty}(\Omega)} \ln \left(1+\|\Delta \omega\|_{L_{2}(\Omega)}\right) \mathcal{P}
$$

in which RHS exhibits dependence on $\mathcal{P}$ involving a smaller exponent than in bounds (10)-(11) when $\|\omega\|_{L_{\infty}(\Omega)}$ is assumed fixed. We remark that, unlike estimates (10)-(12), upper bounds (15) and (17) rely on the control of higher (second) derivatives of vorticity through $\|\triangle \omega\|_{L_{2}(\Omega)}$ and of the vorticity maximum through $\|\omega\|_{L_{\infty}(\Omega)}$. While both these quantities are known to be bounded with respect to time in $2 \mathrm{D}$, they are not necessarily bounded as $\mathcal{P}$ increases, which is the sense of sharpness we are concerned with in this study. In addition, in the absence of a priori bounds on $\|\omega\|_{L_{\infty}(\Omega)}$ in 3D, estimate (16) has a rather different structure from the available finite-time estimate in 3D (table 1). We also emphasize that the presence of the norm $\|\omega\|_{L_{\infty}(\Omega)}$, which is not a smooth function of the vorticity $\omega$, in estimates (15)-(16) would complicate the formulation of the variational optimization problems designed to probe their sharpness (cf. section 3), as these problems would be nonsmooth. On the other hand, we note that finite-time estimate (16) features a milder dependence on the viscosity $\nu$, and hence is likely more optimal with respect to viscosity than bounds (13) and (14). Sharpness of estimates with respect to variations of $\nu$ is however outside the scope of the present study. Some early results concerning the maximum growth of palinstrophy were also obtained by Pouquet et al. (1975), whereas estimates for the rate of growth in the presence of body forcing were studied by Dascaliuc et al. (2010).

Aside from the questions concerning the sharpness of estimates (10)-(12), there is also independent interest in the structure of the vorticity fields leading to the maximum possible palinstrophy production because of their relevance for the enstrophy cascade in 2D turbulence. In fact, various processes related to the stretching of vorticity gradients 
described by equation (8) have already received some attention in the literature (e.g., Protas et al., 1999).

\section{Probing Sharpness of Estimates Using Variational Optimization}

We now go on to discuss how the question of the sharpness of estimates (10), (11) and (12) can be framed in terms of solutions of suitably-defined optimization problems. Analogous questions pertaining to problems in $1 \mathrm{D}$ and $3 \mathrm{D}$, cf. table 1, have already been addressed by Ayala \& Protas (2011) and Lu \& Doering (2008), respectively. As regards estimate $(10)$, the approach consists in finding, for fixed values of $\mathcal{E}=\mathcal{E}_{0}$ and $\mathcal{P}=\mathcal{P}_{0}$, the streamfunction field $\tilde{\psi}_{\mathcal{E}_{0}, \mathcal{P}_{0}}$ which achieves the greatest rate of palinstrophy production $\mathcal{R}_{\mathcal{P}_{0}}\left(\tilde{\psi}_{\mathcal{E}_{0}, \mathcal{P}_{0}}\right)$, and then studying how it depends on the parameters $\mathcal{E}_{0}$ and $\mathcal{P}_{0}$ to see whether or not this dependence follows the predictions of estimate (10) (the use of streamfunction $\psi$, rather than the vorticity or velocity field, as the control variable leads to a simpler formulation of the optimization problem). As regards estimates (11) and (12), the approach is the same, except that, respectively, $\mathcal{K}=\mathcal{K}_{0}$ and $\mathcal{P}$ or just $\mathcal{P}$ are fixed. Thus, we arrive at the following two optimization problems corresponding to estimates (10) and (11)

$$
\begin{aligned}
\tilde{\psi}_{\mathcal{E}_{0}, \mathcal{P}_{0}} & =\underset{\psi \in \mathcal{S}_{\mathcal{E}_{0}, \mathcal{P}_{0}}}{\arg \max } \mathcal{R}_{\mathcal{P}_{0}}(\psi) \\
\mathcal{S}_{\mathcal{E}_{0}, \mathcal{P}_{0}} & =\left\{\psi \in H^{4}(\Omega): \frac{1}{2} \int_{\Omega}(\Delta \psi)^{2} d \Omega=\mathcal{E}_{0}, \frac{1}{2} \int_{\Omega}|\nabla \Delta \psi|^{2} d \Omega=\mathcal{P}_{0}\right\}, \\
\tilde{\psi}_{\mathcal{K}_{0}, \mathcal{P}_{0}} & =\underset{\psi \in \mathcal{S}_{\mathcal{K}_{0}, \mathcal{P}_{0}}}{\arg \max } \mathcal{R}_{\mathcal{P}_{0}}(\psi) \\
\mathcal{S}_{\mathcal{K}_{0}, \mathcal{P}_{0}} & =\left\{\psi \in H^{4}(\Omega): \frac{1}{2} \int_{\Omega}|\nabla \psi|^{2} d \Omega=\mathcal{K}_{0}, \frac{1}{2} \int_{\Omega}|\nabla \Delta \psi|^{2} d \Omega=\mathcal{P}_{0}\right\},
\end{aligned}
$$

where "arg max" denotes "the argument of the maximum" and maximization is performed over the Sobolev space $H^{4}(\Omega)$ of doubly-periodic functions with square-integrable fourthorder derivatives (Adams \& Fournier, 2005). This regularity requirement plays a key role in the solution of optimization problems (18)-(19) as it ensures that the expression for the rate of growth of palinstrophy $\mathcal{R}_{\mathcal{P}_{0}}(\psi)$, cf. (9), is well-defined. We remark that the pairs of constraints in problems (18) and (19) are not in fact independent and must satisfy Poincaré's inequalities, i.e., $\mathcal{E}_{0} \leq(2 \pi)^{-2} \mathcal{P}_{0}$ and $\mathcal{K}_{0} \leq(2 \pi)^{-4} \mathcal{P}_{0}$. The maximization problem corresponding to estimate (12) then takes the form

$$
\begin{aligned}
& \tilde{\psi}_{\mathcal{P}_{0}}=\underset{\psi \in \mathcal{S}_{\mathcal{P}_{0}}}{\arg \max } \mathcal{R}_{\mathcal{P}_{0}}(\psi) \\
& \mathcal{S}_{\mathcal{P}_{0}}=\left\{\psi \in H^{4}(\Omega): \frac{1}{2} \int_{\Omega}|\nabla \Delta \psi|^{2} d \Omega=\mathcal{P}_{0}\right\}
\end{aligned}
$$


in which only one constraint is present (in view of the earlier remark, we note that fixing palinstrophy $\mathcal{P}(\psi)=\mathcal{P}_{0}$ also provides upper bounds, via the aforementioned Poincaré's inequalities, on both enstrophy $\mathcal{E}(\psi)$ and energy $\mathcal{K}(\psi))$. As will be shown in the next section, single-constraint problem (20) is in fact fairly straightforward to solve numerically given the isotropic nature of the constraint. On the other hand, twoconstraint problems (18) and (19) are much harder to solve, since the maximizers are to be sought at the intersection of two nonlinear constraint manifolds which may have a fairly complicated structure, both locally and globally.

We note that, due to the presence of the cubic term in the expression for $\mathcal{R}_{\mathcal{P}_{0}}(\psi)$, cf. (9), optimization problems (18)-(20) may be nonconvex, and hence the presence of multiple local maxima may be expected. We remark here that rescaling the domain $\Omega$ by rational factors will lead to a trivial multiplicity of the optimizing solutions. To demonstrate this, we consider system (3) on a rescaled domain $\Omega_{L}:=[0, L] \times[0, L]$, where $L>0$. The new independent variables become $\boldsymbol{\xi}:=L \mathbf{x} \in \Omega_{L}$ and $\tau:=L^{\beta} t$ for some $\beta \in$ $\mathbb{R}$, whereas the corresponding solution can be expressed as $\Psi(\tau, \boldsymbol{\xi}):=L^{\alpha} \psi(t(\tau), \mathbf{x}(\boldsymbol{\xi}))$ for some $\alpha \in \mathbb{R}$. Transforming system (3) to the new variables, we observe that its form remains unchanged, provided that $\alpha=0$ and $\beta=2$. This shows that $\tilde{\Psi}_{\mathcal{E}_{0}, \mathcal{P}_{0}}:=$ $\tilde{\psi}_{\mathcal{E}_{0}, \mathcal{P}_{0}}(\mathbf{x}(\boldsymbol{\xi}))$ is a solution of optimization problem (18) rescaled to the new domain $\Omega_{L}$. In the particular case when $L=1 / 2,1 / 3, \ldots$ the domain $\Omega_{L}$ is periodically embedded in

$\Omega$. In such situation the maximizers $\widetilde{\Psi}_{\mathcal{E}_{0}, \mathcal{P}_{0}}$ are nothing, but higher-wavenumber copies of the "main" maximizer $\tilde{\psi}_{\mathcal{E}_{0}, \mathcal{P}_{0}}$ and this trivial multiplicity of maximizing solutions will not be separately considered here.

\section{Gradient-Based Solution of Maximization Problems}

In this section we describe key elements of the computational algorithm for the solution of maximization problems stated in section 3. We do this in the spirit of the "optimizethen-discretize" approach (Gunzburger, 2003). Solutions of maximization problems (18), (19) and (20) are characterized by the first-order optimality condition

$$
\mathcal{R}_{\mathcal{P}_{0}}^{\prime}\left(\tilde{\psi} ; \psi^{\prime}\right)+\sum_{i=1}^{m} \lambda_{i} \mathcal{Q}_{i}^{\prime}\left(\tilde{\psi} ; \psi^{\prime}\right)=0 \quad \forall \psi^{\prime} \in H^{4}(\Omega)
$$

where

$$
\begin{aligned}
\mathcal{R}_{\mathcal{P}_{0}}^{\prime}\left(\psi ; \psi^{\prime}\right) & :=\lim _{\epsilon \rightarrow 0}\left[\mathcal{R}_{\mathcal{P}_{0}}\left(\psi+\epsilon \psi^{\prime}\right)-\mathcal{R}_{\mathcal{P}_{0}}(\psi)\right] / \epsilon \quad \text { and } \\
\mathcal{Q}_{i}^{\prime}\left(\psi ; \psi^{\prime}\right) & :=\lim _{\epsilon \rightarrow 0}\left[\mathcal{Q}_{i}\left(\psi+\epsilon \psi^{\prime}\right)-\mathcal{Q}_{i}(\psi)\right] / \epsilon, \quad i=1, \ldots, m
\end{aligned}
$$

are the Gâteaux (directional) differentials (Luenberger, 1969) of, respectively, the objective function and the individual constraints $\mathcal{Q}_{i}(\psi)$ defining the manifolds $\mathcal{S}_{\mathcal{E}_{0}, \mathcal{P}_{0}}, \mathcal{S}_{\mathcal{K}_{0}, \mathcal{P}_{0}}$ and $\mathcal{S}_{\mathcal{P}_{0}}$, cf. (18), (19) and (20). The field $\psi^{\prime}$ represents an arbitrary direction of differentiation in the space $H^{4}(\Omega)$, and $\lambda_{i} \in \mathbb{R}$ are the Lagrange multipliers associated 
with the constraints $\mathcal{Q}_{i}, i=1, \ldots, m(m=2$ for problems (18) and (19), and $m=1$ for problem (20)). We add here that an optimality condition such as (21) could not be easily stated for a variational problem designed to probe the sharpness of estimate (15) with a constraint on $\|\Delta \psi\|_{L_{\infty}(\Omega)}$ because of the nondifferentiability of this constraint.

The maximizer $\tilde{\psi}$ can be found using the following iterative gradient-ascent algorithm which can be interpreted as a discretization of a continuous gradient flow

$$
\begin{aligned}
\tilde{\psi} & =\lim _{n \rightarrow \infty} \psi^{(n)} \\
\psi^{(n+1)} & =\mathbb{P} \mathcal{S}\left(\psi^{(n)}+\tau_{n} \nabla \mathcal{R}_{\mathcal{P}}\left(\psi^{(n)}\right)\right) \\
\psi^{(1)} & =\psi_{0},
\end{aligned}
$$

where $\psi^{(n)}$ is the approximation of the maximizer $\tilde{\psi}$ obtained at the $n$-th iteration, $\psi_{0}$ is the initial guess, $\tau_{n}$ the length of the step and $\mathbb{P} \mathcal{S}: H^{4} \rightarrow \mathcal{S}$ is the projection operator onto the constraint manifold $\mathcal{S}$ (without subscripts, symbol $\mathcal{S}$ denotes a generic manifold). We emphasize that the use of the projection $\mathbb{P} \mathcal{S}$ ensures that, at every step $n$ in optimization iteration (22), the constraint $\psi^{(n)} \in \mathcal{S}$ is satisfied up to machine precision. From the computational point of view, such formulation is in fact preferred to the more standard approach based on Lagrange multipliers and projections onto the tangent space $T \mathcal{S}$ which involve linearization of the constraints and hence result in accumulation of errors.

A key ingredient of algorithm (22) is evaluation of the gradient $\nabla \mathcal{R}_{\mathcal{P}_{0}}$ of objective function $\mathcal{R}_{\mathcal{P}_{0}}(\psi)$, cf. (9), representing its (infinite-dimensional) sensitivity to perturbations of the streamfunction $\psi$. It is essential that the gradient be characterized by the required regularity, namely, $\nabla \mathcal{R}_{\mathcal{P}_{0}}(\psi) \in H^{4}(\Omega)$. This is, in fact, guaranteed by Riesz representation theorem (Luenberger, 1969), applicable because the Gâteaux differential $\mathcal{R}_{\mathcal{P}_{0}}^{\prime}(\psi ; \cdot): H^{4}(\Omega) \rightarrow \mathbb{R}$ is a bounded linear functional on $H^{4}(\Omega)$. Thus, we have

$$
\mathcal{R}_{\mathcal{P}_{0}}^{\prime}\left(\psi ; \psi^{\prime}\right)=\left\langle\nabla \mathcal{R}_{\mathcal{P}_{0}}(\psi), \psi^{\prime}\right\rangle_{H^{4}(\Omega)}=\left\langle\nabla^{L_{2}} \mathcal{R}_{\mathcal{P}_{0}}(\psi), \psi^{\prime}\right\rangle_{L_{2}(\Omega)}
$$

in which the Riesz representers $\nabla \mathcal{R}_{\mathcal{P}_{0}}(\psi)$ and $\nabla^{L_{2}} \mathcal{R}_{\mathcal{P}_{0}}(\psi)$ are the gradients computed with respect to the $H^{4}$ and $L_{2}$ topology, respectively. The corresponding inner products are defined as follows

$$
\begin{aligned}
\forall z_{1}, z_{2} \in L_{2}(\Omega) \quad\left\langle z_{1}, z_{2}\right\rangle_{L_{2}(\Omega)} & =\int_{\Omega} z_{1} z_{2} d \Omega \\
\forall z_{1}, z_{2} \in H^{4}(\Omega) \quad\left\langle z_{1}, z_{2}\right\rangle_{H^{4}(\Omega)} & =\int_{\Omega} z_{1} z_{2}+\ell_{1}^{2} \nabla z_{1} \cdot \nabla z_{2}+\ell_{2}^{4} \Delta z_{1} \Delta z_{2} \\
& +\ell_{3}^{6} \nabla \Delta z_{1} \cdot \nabla \Delta z_{2}+\ell_{4}^{8} \Delta^{2} z_{1} \Delta^{2} z_{2} d \Omega
\end{aligned}
$$

where $\ell_{1}, \ell_{2}, \ell_{3} \geq 0, \ell_{4}>0$ are adjustable parameters with the meaning of lengthscales (Protas et al., 2004). While the $H^{4}$ inner products (25) corresponding to different values of $\ell_{1}, \ell_{2}, \ell_{3}, \ell_{4}$ are mathematically equivalent (in the sense of norm equivalence, 
Luenberger (1969)), these parameters will play a role in tuning the performance of the discrete optimization algorithm discussed in section 6 . We remark that while the $H^{4}$ gradient is used exclusively in the actual computations, cf. (22), the $L_{2}$ gradient is computed first as an intermediate step. Calculating the Gâteaux differential of $\mathcal{R}_{\mathcal{P}_{0}}(\psi)$ and identifying it with the $L_{2}$ inner product (24) we thus obtain

$$
\begin{aligned}
\mathcal{R}_{\mathcal{P}_{0}}^{\prime}\left(\psi ; \psi^{\prime}\right) & =\int_{\Omega}\left[\Delta^{2} J(\Delta \psi, \psi)+\Delta J\left(\psi, \Delta^{2} \psi\right)+J\left(\Delta^{2} \psi, \Delta \psi\right)-2 \nu \Delta^{4} \psi\right] \psi^{\prime} d \Omega \\
& =\left\langle\nabla^{L_{2}} \mathcal{R}_{\mathcal{P}_{0}}(\psi), \psi^{\prime}\right\rangle_{L_{2}(\Omega)}
\end{aligned}
$$

from which it follows that

$$
\nabla^{L_{2}} \mathcal{R}_{\mathcal{P}_{0}}(\psi)=\Delta^{2} J(\Delta \psi, \psi)+\Delta J\left(\psi, \Delta^{2} \psi\right)+J\left(\Delta^{2} \psi, \Delta \psi\right)-2 \nu \Delta^{4} \psi
$$

Identifying the left-hand side (LHS) of (26) with the $H^{4}$ inner product (25), integrating by parts and using (27), we obtain the required $H^{4}$ gradient as the solution of the following elliptic boundary-value problem

$$
\left[\mathrm{Id}-\ell_{1}^{2} \Delta+\ell_{2}^{4} \Delta^{2}-\ell_{3}^{6} \Delta^{3}+\ell_{4}^{8} \Delta^{4}\right] \nabla \mathcal{R}_{\mathcal{P}_{0}}=\nabla^{L_{2}} \mathcal{R}_{\mathcal{P}_{0}} \quad \text { in } \Omega
$$

Periodic Boundary Conditions.

As shown by Protas et al. (2004), extraction of gradients in spaces of smoother functions such as $H^{4}(\Omega)$ can be interpreted as low-pass filtering of the $L_{2}$ gradients with parameters $\ell_{1}, \ell_{2}, \ell_{3}, \ell_{4}$ acting as cut-off length-scales.

The step size $\tau_{n}$ in algorithm (22) is calculated as

$$
\tau_{n}=\underset{\tau>0}{\arg \max }\left\{\mathcal{R}_{\mathcal{P}}\left[\mathbb{P} \mathcal{S}\left(\psi^{(n)}+\tau \nabla \mathcal{R}_{\mathcal{P}}\left(\psi^{(n)}\right)\right)\right]\right\}
$$

which is done using a derivative-free line search algorithm (Ruszczyński, 2006). Equation (29) can be interpreted as a modification of a standard line search method where the optimization is performed following an arc (a geodesic) lying on the constraint manifold $\mathcal{S}$, rather than a straight line. This approach was already successfully employed to solve a similar problem in Ayala \& Protas (2011). The projection $\phi \mapsto \mathbb{P} \mathcal{S}(\phi)$ for some $\phi \in H^{4}(\Omega)$ is calculated by solving an optimization subproblem with form depending on the type of the constraint as follows.

- Single Constraint: problem (20) involves the constraint manifold

$$
\mathcal{S}_{\mathcal{P}_{0}}=\left\{\phi \in H^{4}(\Omega): \frac{1}{2} \int_{\Omega}|\nabla \Delta \phi|^{2} d \Omega=\mathcal{P}_{0}\right\} .
$$

Then, the projection operator $\mathbb{P} \mathcal{S}_{\mathcal{P}_{0}}$ is defined as the renormalization

$$
\mathbb{P} \mathcal{S}_{\mathcal{P}_{0}}(\phi)=\sqrt{\frac{\mathcal{P}_{0}}{\mathcal{P}(\phi)}} \phi .
$$


- $\left(\mathcal{E}_{0}, \mathcal{P}_{0}\right)$-Constraint: problem (18) involves the constraint manifold

$$
\mathcal{S}_{\mathcal{E}_{0}, \mathcal{P}_{0}}=\left\{\phi \in H^{4}(\Omega): \frac{1}{2} \int_{\Omega}(\Delta \phi)^{2} d \Omega=\mathcal{E}_{0}, \frac{1}{2} \int_{\Omega}|\nabla \Delta \phi|^{2} d \Omega=\mathcal{P}_{0}\right\} .
$$

Then, the projection $\phi \mapsto \mathbb{P} \mathcal{S}_{\mathcal{E}_{0}, \mathcal{P}_{0}}(\phi)$ is computed as $\mathbb{P} \mathcal{S}_{\mathcal{E}_{0}, \mathcal{P}_{0}}(\phi)=\lim _{k \rightarrow \infty} \varphi^{(k)}$, where

$$
\begin{aligned}
\varphi^{(k+1)} & =\varphi^{(k)}-\tau_{k} \nabla \mathcal{Q}\left(\varphi^{(k)}\right), \quad k=1,2, \ldots, \\
\varphi^{(1)} & =\phi
\end{aligned}
$$

in which $\mathcal{Q}(\varphi)=\frac{1}{2}\left[\mathcal{P}(\varphi)-\mathcal{P}_{0}\right]^{2}$ and $\nabla \mathcal{Q}(\varphi)$ is the corresponding gradient. That is, the projection onto $\mathcal{S}_{\mathcal{E}_{0}, \mathcal{P}_{0}}$ is obtained by solving a single-constraint optimization problem with cost functional $\mathcal{Q}(\varphi)$ penalizing the deviation from the second constraint and the first constraint enforced using (31).

- $\left(\mathcal{K}_{0}, \mathcal{P}_{0}\right)$-Constraint: problem (19) involves the constraint manifold

$$
\mathcal{S}_{\mathcal{K}_{0}, \mathcal{P}_{0}}=\left\{\phi \in H^{4}(\Omega): \frac{1}{2} \int_{\Omega}|\nabla \phi|^{2} d \Omega=\mathcal{K}_{0}, \frac{1}{2} \int_{\Omega}|\nabla \Delta \phi|^{2} d \Omega=\mathcal{P}_{0}\right\} .
$$

In analogy to the $\left(\mathcal{E}_{0}, \mathcal{P}_{0}\right)$-constraint, the projection onto $\mathcal{S}_{\mathcal{K}_{0}, \mathcal{P}_{0}}$ is obtained by solving a single-constraint optimization problem of the type (33) with cost functional $\mathcal{Q}(\varphi)=\frac{1}{2}\left[\mathcal{K}(\varphi)-\mathcal{K}_{0}\right]^{2}$.

We add that, since none of the manifolds defined in (30), (32) and (34) has the structure of a linear space, the projections defined above are not orthogonal.

Families of maximizers parameterized by their palinstrophy $\mathcal{P}_{0}$ are found by solving problems (18), (19) and (20) for values of $\mathcal{P}_{0}$ progressively incremented or decremented by $\pm \Delta \mathcal{P}_{0}$ and using the previously obtained maximizer $\tilde{\psi}_{\mathcal{P}_{0}}$ as the initial guess $\psi_{0}$ for $\tilde{\psi}_{\mathcal{P}_{0} \pm \Delta \mathcal{P}_{0}}$ in (22). In order to carry out an exhaustive search for all possible maximizing fields, this process was initiated in a variety of ways, including different random fields and closed-form solutions to the limiting problems described in the next section.

\section{Solution of the Maximization Problems in the Limit of Small Palinstrophies}

In this section we investigate the structure of the maximizing solutions in the limiting cases when $\mathcal{P}_{0} \rightarrow 0$ in $(20), \mathcal{P}_{0} \rightarrow(2 \pi)^{2} \mathcal{E}_{0}$ in (18) and $\mathcal{P}_{0} \rightarrow(2 \pi)^{4} \mathcal{K}_{0}$ in (19). Motivated by the properties of the vortex states found numerically for large values of $\mathcal{P}_{0}$ (see section 6 ), we will consider optimal vorticity distributions in the form of periodic vortex lattices with 2-fold rotational symmetry, i.e., possessing the property $\psi(x, y)=-\psi(y,-x)$. 
We begin the discussion by analyzing the single-constraint optimization problem (20) in the limit $\mathcal{P}_{0} \rightarrow 0$. The Euler-Lagrange equation characterizing the solutions of this problem is, cf. (9),

$$
\begin{aligned}
\mathcal{G}(\psi)+2 \nu \Delta^{4} \psi+\lambda \Delta^{3} \psi & =0 \\
\frac{1}{2} \int_{\Omega}(\nabla \Delta \psi)^{2} d \Omega & =\mathcal{P}_{0},
\end{aligned}
$$

where $\lambda \in \mathbb{R}$ is the Lagrange multiplier associated with constraint (35b), equation (35a) is subject to the doubly-periodic boundary conditions and we denoted $\mathcal{G}(\psi):=$ $\Delta^{2} J(\Delta \psi, \psi)+\Delta J\left(\psi, \Delta^{2} \psi\right)+J\left(\Delta^{2} \psi, \Delta \psi\right)$. In order to obtain insights about the behavior of solutions $(\psi, \lambda)$ of $(35)$ in the limit $\mathcal{P}_{0} \rightarrow 0$, we use the following series expansion

$$
\begin{aligned}
& \psi=\psi_{0}+\mathcal{P}_{0}^{1 / 2} \psi_{1}+\mathcal{P}_{0}^{1} \psi_{2}+O\left(\mathcal{P}_{0}^{3 / 2}\right), \\
& \lambda=\lambda_{0}+\mathcal{P}_{0}^{1 / 2} \lambda_{1}+\mathcal{P}_{0}^{1} \lambda_{2}+O\left(\mathcal{P}_{0}^{3 / 2}\right) .
\end{aligned}
$$

Introducing ansatz (36) in (35) and collecting terms proportional to different powers of $\mathcal{P}_{0}^{1 / 2}$, we obtain at the leading order

$$
\begin{aligned}
\mathcal{P}_{0}^{0}: \quad & 2 \nu \Delta^{4} \psi_{0}+\lambda_{0} \Delta^{3} \psi_{0}=-\mathcal{G}\left(\psi_{0}\right), \\
\frac{1}{2} \int_{\Omega}\left(\nabla \Delta \psi_{0}\right)^{2} d \Omega & =0,
\end{aligned}
$$

from which it follows immediately that $\psi_{0} \equiv 0$. Using this result, at the next order we have

$$
\begin{aligned}
\mathcal{P}_{0}^{1 / 2}: \quad & 2 \nu \Delta^{4} \psi_{1}+\lambda_{0} \Delta^{3} \psi_{1}=0 \\
\frac{1}{2} \int_{\Omega}\left(\nabla \Delta \psi_{1}\right)^{2} d \Omega & =1
\end{aligned}
$$

where we note that the vanishing of the contribution from $\mathcal{G}(\psi)$ in $(38 \mathrm{a})$ is due to $\psi_{0}$ being identically zero. While continuing this process might lead to some interesting insights, for our present purposes it is in fact sufficient to truncate expansions (36) at the order $O\left(\mathcal{P}_{0}\right)$. The corresponding approximation of our objective function (9) thus becomes

$$
\mathcal{R}_{\mathcal{P}_{0}}=-\mathcal{P}_{0} \nu \int_{\Omega}\left(\Delta \psi_{1}\right)^{2} d \Omega+O\left(\mathcal{P}_{0}^{3 / 2}\right)
$$

As regards problem (38) defining $\left(\psi_{1}, \lambda_{0}\right)$, we note that, since for zero-mean functions defined on doubly-periodic domains, $\operatorname{Ker}\left(\Delta^{3}\right)=\{0\}$, equation (38a) becomes an eigenvalue problem $\Delta \psi_{1}=\lambda_{0}^{\prime} \psi_{1}$, where $\lambda_{0}^{\prime}:=-\lambda_{0} /(2 \nu)<0$. It can be shown via direct calculation that $\mathcal{R}_{\mathcal{P}_{0}} \approx 2 \nu \lambda_{0}^{\prime} \mathcal{P}_{0}$ as $\mathcal{P}_{0} \rightarrow 0$, cf. (39), and we are therefore interested in the eigenfunctions associated with the largest, i.e., the least negative, eigenvalues. There are two distinct possibilities corresponding to different arrangements of vortices with 2 -fold rotational symmetry in the domain $\Omega$, namely (cf. figure 1 ), 


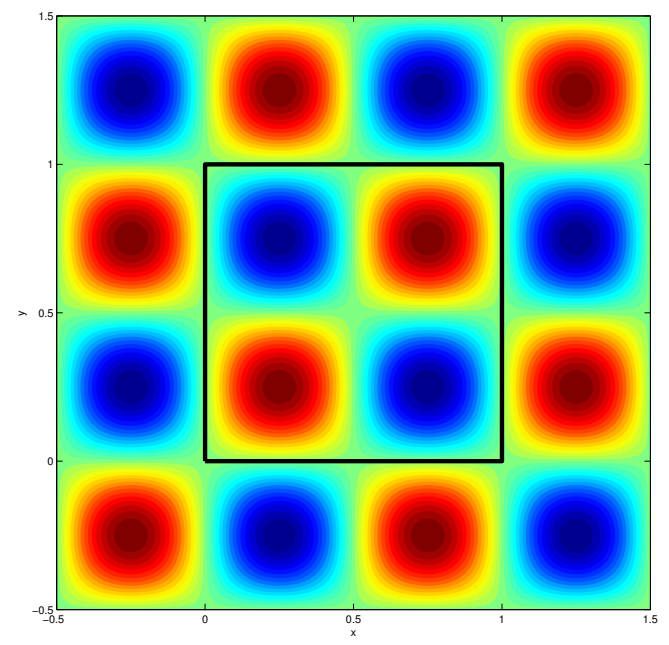

(a)

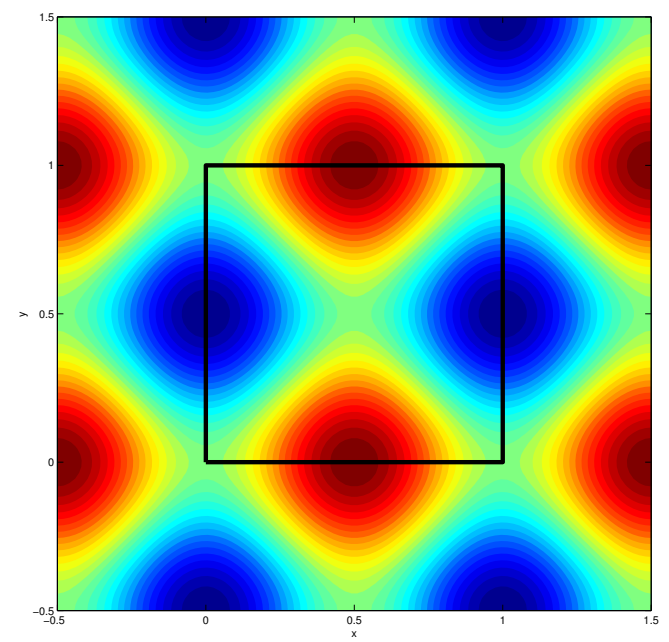

(b)

Figure 1: Vorticity fields characterized by (a) aligned and (b) staggered arrangement of vortex cells obtained as the solutions of maximization problem (20) in the limit $\mathcal{P}_{0} \rightarrow 0$. They are given by, respectively, expressions (40) with $p=1$ and (42) with $p=1 / 2$. The boundary of the domain $\Omega$ is marked with the black solid line.

- aligned arrangement where

$$
\psi_{1, a}(x, y)=\frac{1}{4} \sin (2 \pi p x) \sin (2 \pi q y), \quad p=1,2, \ldots
$$

with the eigenvalue $\lambda_{0}^{\prime}=-8 \pi^{2} p^{2}$ which is maximized for $p=1$ resulting in

$$
\mathcal{R}_{\mathcal{P}_{0}} \approx-16 \pi^{2} \nu \mathcal{P}_{0}
$$

- staggered arrangement where

$$
\psi_{1, s}(x, y)=\frac{1}{4} \sin [2 \pi p(x+y)] \sin [2 \pi p(x-y)], \quad p=1 / 2,1,3 / 2, \ldots
$$

with the eigenvalue $\lambda_{0}^{\prime}=-16 \pi^{2} p^{2}$ which is maximized for $p=1 / 2$ resulting in

$$
\mathcal{R}_{\mathcal{P}_{0}} \approx-8 \pi^{2} \nu \mathcal{P}_{0}
$$

We therefore conclude that, while $\mathcal{R}_{\mathcal{P}_{0}}\left(\psi_{1}\right)$ is negative-definite for both arrangements, it assumes larger (i.e., less negative) values for the staggered configuration. To prove that $\psi_{1}$ is indeed a local maximizer of $\mathcal{R}_{\mathcal{P}_{0}}(\psi)$ in the limit $\mathcal{P}_{0} \rightarrow 0$, rather than just a saddle point, it would be necessary to demonstrate the negative definiteness of the 
Hessian of $\mathcal{R}_{\mathcal{P}_{0}}(\psi)$ at $\psi_{1}$. This, however, becomes technically complicated and we will not study it here. Computational results presented in section 6 provide evidence that indeed the maximizers of the single-constraint problem (20) approach the field $\mathcal{P}_{0}^{1 / 2} \psi_{1}$ in the limit $\mathcal{P}_{0} \rightarrow 0$. As regards the negativity of $\mathcal{R}_{\mathcal{P}_{0}}\left(\tilde{\psi}_{\mathcal{P}_{0}}\right)$ in this limit, we remark that $J(\varphi, \Delta \varphi) \equiv 0$ when $\varphi$ is an eigenfunction of the Laplacian and then the cubic part of $\mathcal{R}_{\mathcal{P}_{0}}\left(\tilde{\psi}_{\mathcal{P}_{0}}\right)$ vanishes leaving just the dissipative term.

As regards the two-constraint problems (18) and (19) in the respective limits $\mathcal{P}_{0} \rightarrow$ $(2 \pi)^{2} \mathcal{E}_{0}$ and $\mathcal{P}_{0} \rightarrow(2 \pi)^{4} \mathcal{K}_{0}$, we note that, as the leading eigenfunction of the Laplacian, the maximizer $\psi_{1, s}$ defined in (42) saturates Poincaré's inequality, i.e., $\mathcal{P}\left(\psi_{1, s}\right)=$ $(2 \pi)^{2} \mathcal{E}\left(\psi_{1, s}\right)$. This means that second constraints $\mathcal{E}(\psi)=\mathcal{E}_{0}$ and $\mathcal{K}(\psi)=\mathcal{K}_{0}$ are satisfied automatically by $\psi_{1, s}$ and therefore need not be enforced through the introduction of another Lagrange multiplier. This allows us to conclude that $\psi_{1, s}$ is also the solution of the limiting forms of two-constraint optimization problems (18) and (19).

\section{Computational Results}

In the two subsections below we describe the results obtained from the numerical solution of optimization problems (20), (18) and (19) using the computational approaches described in section 4 for a broad range of constraint parameters $\mathcal{P}_{0},\left(\mathcal{E}_{0}, \mathcal{P}_{0}\right)$ and $\left(\mathcal{K}_{0}, \mathcal{P}_{0}\right)$. In subsection 6.3 we discuss how the palinstrophy growth in finite time corresponding to the instantaneous maximizers found in sections 6.1 and 6.2 used as the initial data compares with the available finite-time estimates.

In the calculations described in sections 6.1 and 6.2 the key element is the evaluation of the gradient $\nabla \mathcal{R}_{\mathcal{P}_{0}}(\psi)$, first in the $L_{2}$ and then in the $H^{4}$ topology, cf. (27)-(28), which is done using a pseudospectral Fourier-Galerkin technique with standard dealiasing. The resolution varied from $128^{2}$ to $1024^{2}$ grid points in the low-palinstrophy and highpalinstrophy cases, respectively. Convergence of all calculations with respect to the grid refinement was carefully verified. The rate of convergence of discrete gradient flow (22) depends, among other factors, on the values of the length-scale parameters characterizing the Sobolev inner product (25). Based on our extensive numerical test, we used $\ell_{1}=\ell_{2}=0, \ell_{3} \in\left[10^{-2}, 10^{-1}\right]$ and $\ell_{4} \in\left[10^{-4}, 10^{-2}\right]$ (with smaller values of $\ell_{3}$ and $\ell_{4}$ corresponding to higher numerical resolutions). We remark that, given the equivalence of the inner products (25) corresponding to different values of $\ell_{1}, \ell_{2}, \ell_{3}, \ell_{4}$, these choices do not affect the maximizers found, but only how rapidly they are approached by algorithm (22). In all calculations the value of the viscosity coefficient was $\nu=10^{-3}$.

As regards the results for the time-dependent problem presented in section 6.3, we used a numerical approach combining a standard pseudospectral discretization in space with the Krylov subspace method described by Edwards et al. (1994) for the time discretization to numerically solve system (3) for the vorticity evolution. As regards the initial data $\omega_{0}$, it was chosen as the vorticity corresponding to the solutions of optimization problems (18), (19) and (20), i.e., $-\Delta \tilde{\psi}_{\mathcal{E}_{0}, \mathcal{P}_{0}},-\Delta \tilde{\psi}_{\mathcal{K}_{0}, \mathcal{P}_{0}}$ and $-\Delta \tilde{\psi}_{\mathcal{P}_{0}}$. In these simulations, the resolution varied from $512^{2}$ to $4096^{2}$ grid points depending on the char- 
acteristic length scales of the initial data which ensured that all calculations were wellresolved. The length of the time window over which the problem was solved was chosen for all initial data long enough to capture the initial amplification of the palinstrophy followed by its viscous decay. Power-law exponents mentioned below were computed by fitting a linear polynomial to the $\log _{10}(d \mathcal{P} / d t)$ versus $\log _{10}\left(\mathcal{P}_{0}\right)$ relationship.

\subsection{Optimization Problems with a Single Constraint on $\mathcal{P}_{0}$}

In this section we discuss solutions of the single-constraint optimization problem (20) with the goal of assessing the sharpness of estimate (12). In figures 2(a) and 2(b) we show the maximum palinstrophy rate of growth $\mathcal{R}_{\mathcal{P}_{0}}\left(\tilde{\psi}_{\mathcal{P}_{0}}\right)$ obtained, respectively, for small and large values of $\mathcal{P}_{0}$. We note the presence of two distinct branches of maximizing solutions, and the corresponding vorticity fields $-\Delta \tilde{\psi}_{\mathcal{P}_{0}}$ are shown in figures $2(\mathrm{c}, \mathrm{d}, \mathrm{e})$ and $2(\mathrm{f}, \mathrm{g}, \mathrm{h})$. The two branches arise, via continuation with respect to parameter $\mathcal{P}_{0}$, from the two limiting maximizers discussed in section 5 and characterized by the staggered and aligned arrangement of the vortex cells (cf. figure 1), with the latter case always giving a larger value of $\mathcal{R}_{\mathcal{P}_{0}}\left(\tilde{\psi}_{\mathcal{P}_{0}}\right)$ for a given $\mathcal{P}_{0}$. The localized vortex structure present in the field shown in figure $2(\mathrm{e})$ is magnified in figure 6(a). In agreement with the discussion in section 5 , for small $\mathcal{P}_{0}$ the values of the maximum palinstrophy rate of growth $\mathcal{R}_{\mathcal{P}_{0}}\left(\tilde{\psi}_{\mathcal{P}_{0}}\right)$ on both branches are negative and in the limit $\mathcal{P}_{0} \rightarrow 0, \mathcal{R}_{\mathcal{P}_{0}}\left(\tilde{\psi}_{\mathcal{P}_{0}}\right) \sim-8 \pi^{2} \nu \mathcal{P}_{0}$ and $\mathcal{R}_{\mathcal{P}_{0}}\left(\tilde{\psi}_{\mathcal{P}_{0}}\right) \sim-16 \pi^{2} \nu \mathcal{P}_{0}$ in the two cases (figure $\left.2(\mathrm{a})\right)$. On the other hand, in figure 2(b) we see that for large values of $\mathcal{P}_{0}$ the maximum rate of growth $\mathcal{R}_{\mathcal{P}_{0}}\left(\tilde{\psi}_{\mathcal{P}_{0}}\right)$ exhibits a clear power-law behavior with respect to $\mathcal{P}_{0}$ for both branches. In both cases the exponent is $1.57 \pm 0.05$ which is less than the exponent 2 appearing in estimate (12), cf. table 2 .

\subsection{Optimization Problems with Two Constraints on $\left(\mathcal{E}_{0}, \mathcal{P}_{0}\right)$ and $\left(\mathcal{K}_{0}, \mathcal{P}_{0}\right)$}

We now discuss solutions of two-constraint optimization problems (18) and (19). In order to use these solutions to assess the sharpness of estimates (18) and (19), in both cases we present the results by fixing one of the constrained quantities, $\mathcal{E}_{0}$ or $\mathcal{K}_{0}$, and then studying the maximum rate of growth of palinstrophy $\mathcal{R}_{\mathcal{P}_{0}}\left(\tilde{\psi}_{\mathcal{E}_{0}, \mathcal{P}_{0}}\right)$ and $\mathcal{R}_{\mathcal{P}_{0}}\left(\tilde{\psi}_{\mathcal{K}_{0}, \mathcal{P}_{0}}\right)$ as a function of the palinstrophy $\mathcal{P}_{0}$ which is allowed to vary over several orders of magnitude. The results are shown in figure $3(\mathrm{a})$ for $\mathcal{E}_{0}=100$ and in figure 4 (a) for $\mathcal{K}_{0}=10$, each of which features two distinct solution branches representing the local maximizers. In both cases the values of the palinstrophy $\mathcal{P}_{0}$ for which the maximizing solutions are found are bounded from below by Poincaré's inequalities. In figures 3(c)-(h) and 4(c)-(h) we show the vorticity fields $-\Delta \tilde{\psi}_{\mathcal{E}_{0}, \mathcal{P}_{0}}$ and $-\Delta \tilde{\psi}_{\mathcal{K}_{0}, \mathcal{P}_{0}}$ corresponding to each of the two branches and different values of palinstrophy. We also remark that, as predicted in section 5 , for values of $\mathcal{P}_{0}$ approaching the Poincaré limit, respectively, $\mathcal{P}_{c}=(2 \pi)^{2} \mathcal{E}_{0}$ in the $\left(\mathcal{E}_{0}, \mathcal{P}_{0}\right)$-constrained problem and $\mathcal{P}_{c}=(2 \pi)^{4} \mathcal{K}_{0}$ in the $\left(\mathcal{K}_{0}, \mathcal{P}_{0}\right)$-constrained problem, the maximizers $\tilde{\psi}_{\mathcal{E}_{0}, \mathcal{P}_{0}}$ and $\tilde{\psi}_{\mathcal{K}_{0}, \mathcal{P}_{0}}$ approach the Laplacian eigenfunctions given in (40) and (42), and the pairs of branches shown in Figures 3(a) and 4(a) correspond to the fields with the aligned and 


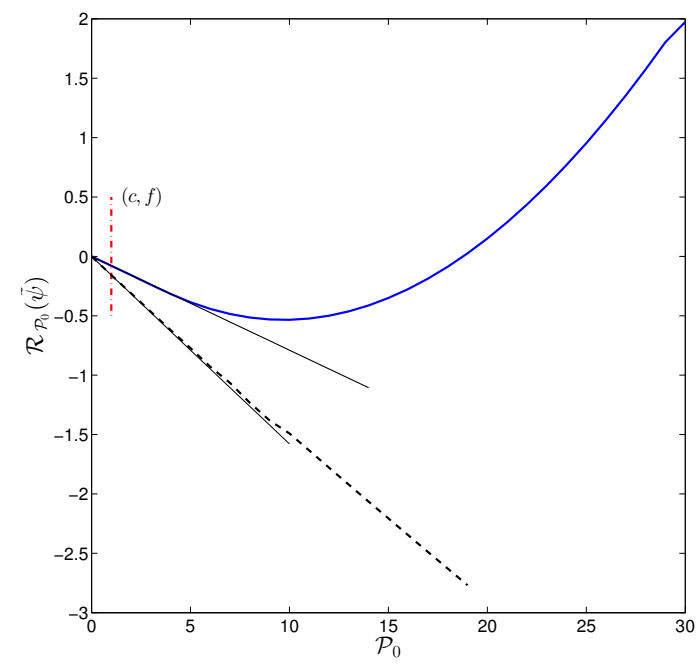

(a)

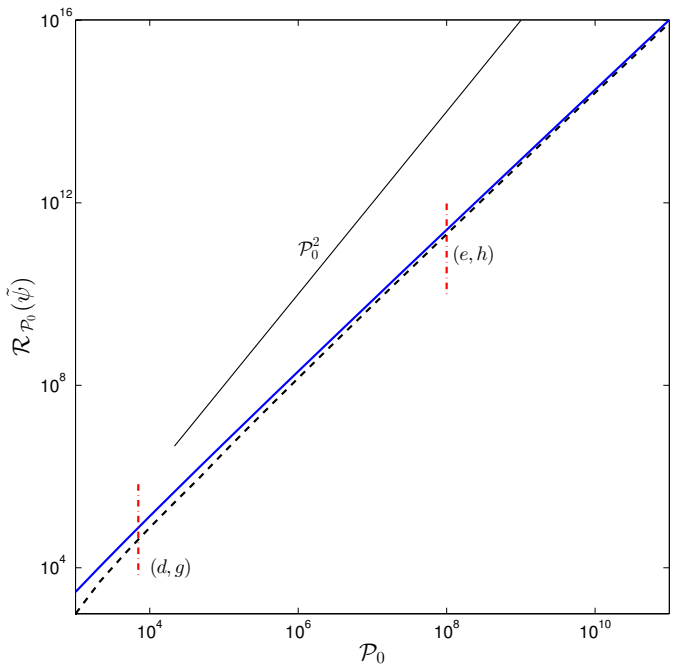

(b)

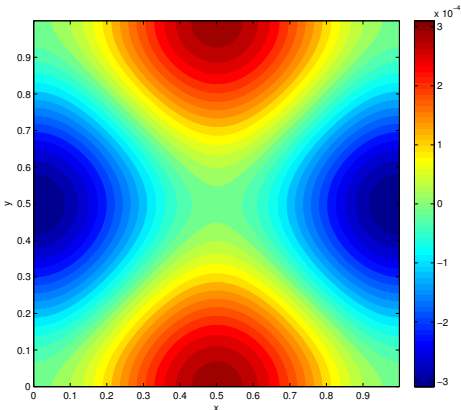

(c)

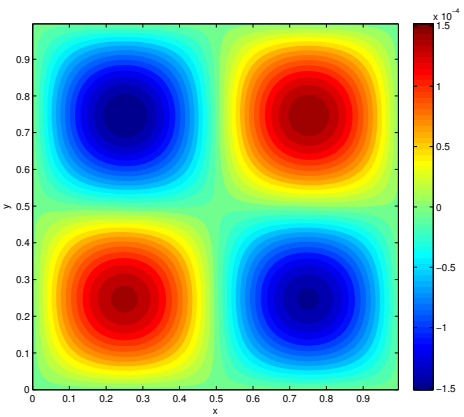

(f)

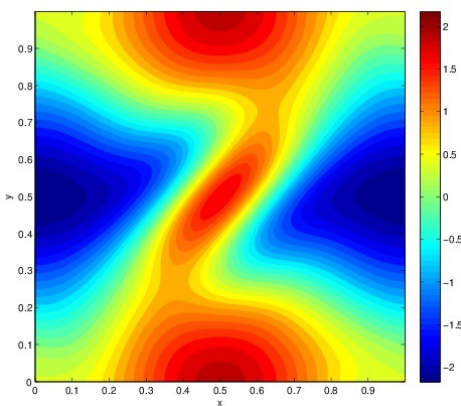

(d)

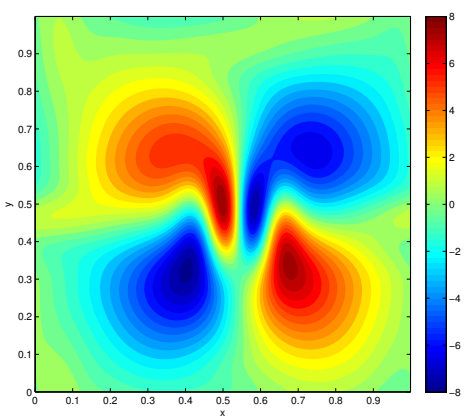

(g)

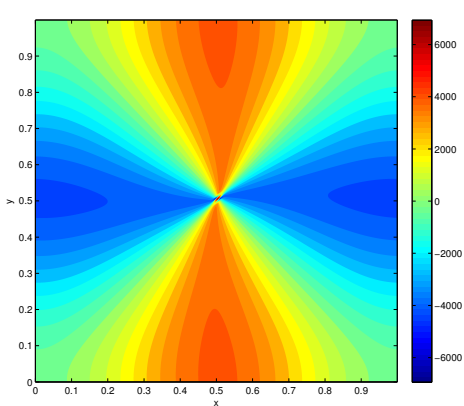

(e)

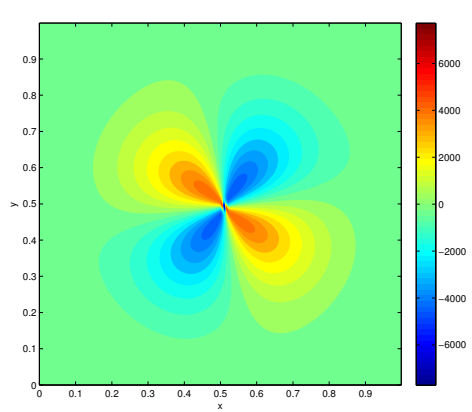

(h)

Figure 2: Dependence of the maximum palinstrophy rate of growth $\mathcal{R}_{\mathcal{P}_{0}}\left(\tilde{\psi}_{\mathcal{P}_{0}}\right)$ on $\mathcal{P}_{0}$ for (a) small $\mathcal{P}_{0}$ and (b) large $\mathcal{P}_{0} ;(\mathrm{c}-\mathrm{h})$ optimal vortex states corresponding to the branch with (c-e) staggered and $(\mathrm{f}-\mathrm{h})$ aligned arrangement of the vortex cells for the values of $\mathcal{P}_{0}$ marked with short vertical lines in figures (a) and (b); since the maximizing vortex states corresponding to the lower branch proved very difficult to compute accurately for intermediate values of $\mathcal{P}_{0}$, this branch is not complete in figure (a). 
staggered arrangements of the "vortex cells", cf. figure 1. The localized vortex structures present in the fields shown in figures $3(\mathrm{e})$ and $4(\mathrm{e})$ are magnified in figures $6(\mathrm{~b}, \mathrm{c})$. In figures $3(\mathrm{~b})$ and $4(\mathrm{~b})$ we present the maximum rate of growth of palinstrophy for a few different values of the first constraint, namely $\mathcal{E}_{0}=10^{2}, 10^{3}, 10^{4}$ and $\mathcal{K}_{0}=10^{0}, 10^{1}, 10^{2}$. For clarity, in these figures we only show the branches with higher values of $\mathcal{R}_{\mathcal{P}_{0}}$ which in both cases correspond to the maximizing fields with staggered vortex cells.

As is evident from figures $3(\mathrm{a}, \mathrm{b})$ and $4(\mathrm{a}, \mathrm{b})$, the two two-constraint problems lead to very different behavior of the maximum rate of growth of palinstrophy when $\mathcal{P}_{0} \rightarrow \infty$. In the case with the $\left(\mathcal{K}_{0}, \mathcal{P}_{0}\right)$-constraint it exhibits a clear power-law characterized by

$$
\frac{d \mathcal{P}}{d t} \sim \mathcal{P}_{0}^{1.49 \pm 0.02}
$$

which is consistent with estimate (11), thereby confirming its sharpness. On the other hand, the case with the $\left(\mathcal{E}_{0}, \mathcal{P}_{0}\right)$-constraint reveals a sharp decrease of $d \mathcal{P} / d t$ observed for large values of $\mathcal{P}_{0}$ regardless of the value of $\mathcal{E}_{0}$. It should be clarified, however, that this does not mean that the branches cannot be continued, but only that for sufficiently large values of $\mathcal{P}_{0}$ the corresponding values of $d \mathcal{P} / d t$ are negative and thus are not shown in a log-log plot. We emphasize that this behavior is in fact consistent with estimate (10) in which the increase of $d \mathcal{P} / d t$ is at large values of $\mathcal{P}_{0}$ limited by the negative-definite quadratic term. As shown in figure $3(\mathrm{a})$, this behavior is qualitatively captured by the dependence of $\mathcal{R}_{\mathcal{P}_{0}}\left(\tilde{\psi}_{\mathcal{E}_{0}, \mathcal{P}_{0}}\right)$ on $\mathcal{P}_{0}$.

\subsection{Palinstrophy Growth in Finite Time}

We consider solutions of Navier-Stokes system (3) with the following initial data

$$
\begin{aligned}
\text { (i) } \omega_{0} & =-\Delta \tilde{\psi}_{\mathcal{P}_{0}}, \\
\text { (ii) } \omega_{0} & =-\Delta \tilde{\psi}_{\mathcal{K}_{0}, \mathcal{P}_{0}}, \\
\text { (iii) } \omega_{0} & =-\Delta \tilde{\psi}_{\mathcal{E}_{0}, \mathcal{P}_{0}} .
\end{aligned}
$$

To obtain insights about the sharpness of finite-time estimates (13) and (14), we are interested in the maximum palinstrophy attained over time $\mathcal{P}_{\max }:=\max _{t>0} \mathcal{P}(t)$ and its increment with respect to the initial value $\delta \mathcal{P}:=\mathcal{P}_{\max }-\mathcal{P}_{0}$ as functions of the initial enstrophy $\mathcal{E}_{0}$ and palinstrophy $\mathcal{P}_{0}$ (the reason for studying $\delta \mathcal{P}$ is that the term $\mathcal{P}_{0}$ may mask the behavior of the other term on the RHS in (13) if it should also scale with an exponent close to the unity). Figure 5 (a) shows $\delta \mathcal{P}$ for each case (i)-(iii) as a function of $\mathcal{P}_{0}$ with $\mathcal{K}_{0}=10$ in case (ii) and $\mathcal{E}_{0}=10^{3}$ in case (iii). These results exhibit a power-law behavior in cases (i) and (ii), whereas in case (iii) a sharp decrease of $\delta \mathcal{P}$ is observed as $\mathcal{P}_{0} \rightarrow \infty$. The power laws for cases (i) and (ii) are

$$
\begin{aligned}
\text { (i) } \delta \mathcal{P} & \sim \mathcal{P}_{0}^{1.13 \pm 0.03} \\
\text { (ii) } \delta \mathcal{P} & \sim \mathcal{P}_{0}^{1.05 \pm 0.09}
\end{aligned}
$$




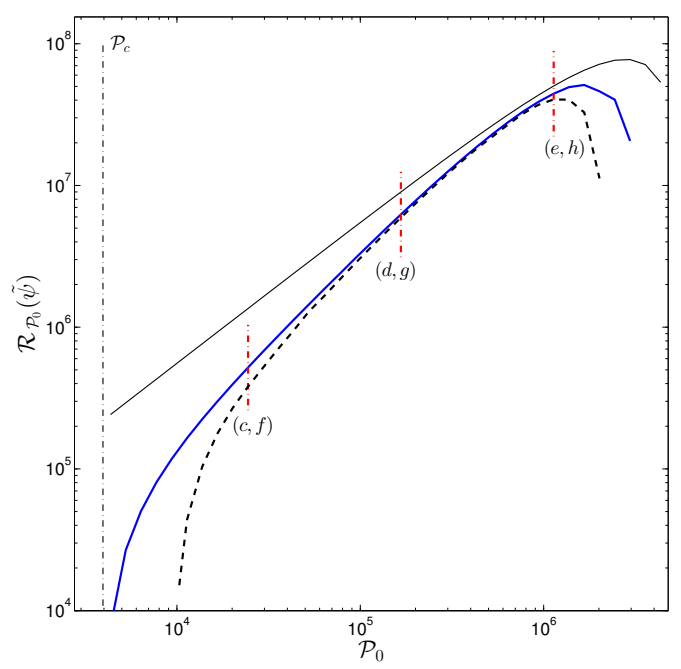

(a)

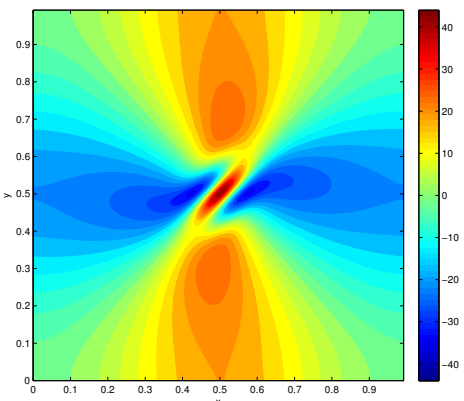

(c)

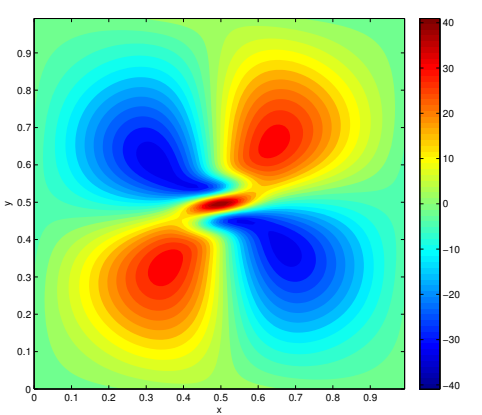

(f)

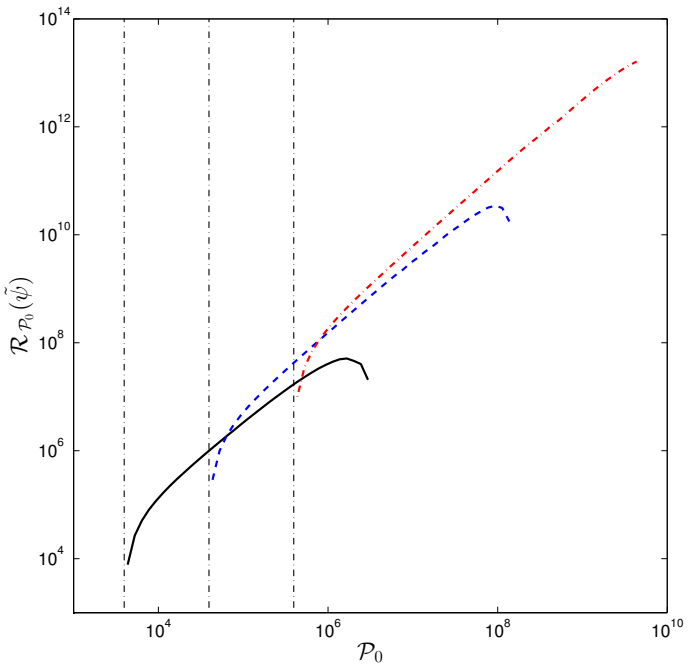

(b)

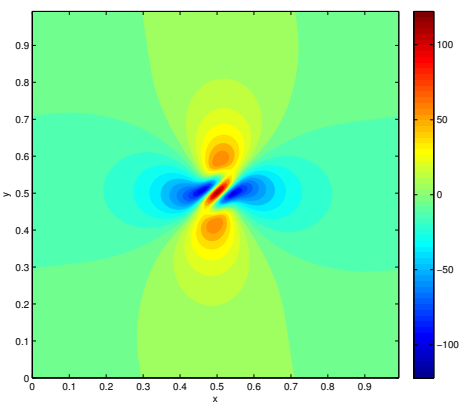

(d)

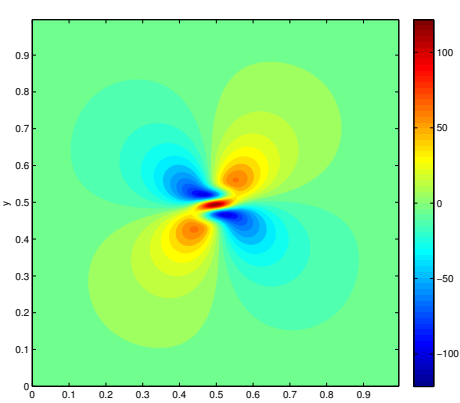

$(\mathrm{g})$

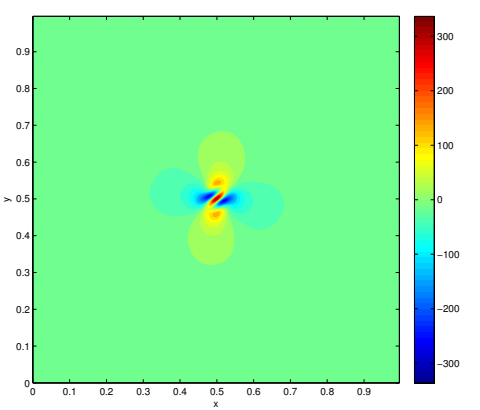

(e)

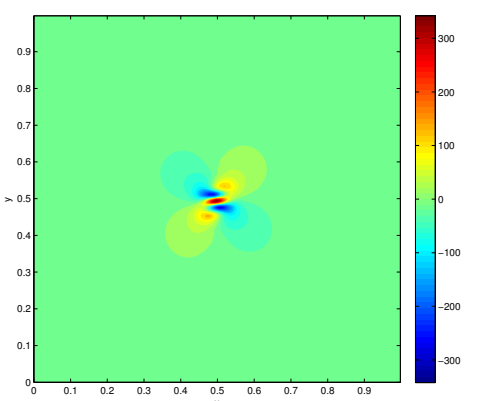

(h)

Figure 3: Dependence of the maximum palinstrophy rate of growth $\mathcal{R}_{\mathcal{P}_{0}}\left(\tilde{\psi}_{\mathcal{E}_{0}, \mathcal{P}_{0}}\right)$ on $\mathcal{P}_{0}$ for (a) $\mathcal{E}_{0}=100$ and (b) $\mathcal{E}_{0}=10^{2}, 10^{3}$ and $10^{4}$. Figure (a) shows both solution branches, whereas figure (b) only the ones with larger values of $\mathcal{R}_{\mathcal{P}_{0}}$. Optimal vortex states corresponding to the two branches are shown in figures (c-e) and ( $\mathrm{f}-\mathrm{h})$ for the following palinstrophy values: $(\mathrm{c}, \mathrm{f}) \mathcal{P}_{0} \approx 10 \mathcal{P}_{c},(\mathrm{~d}, \mathrm{~g}) \mathcal{P}_{0} \approx 10^{2} \mathcal{P}_{c}$ and $(\mathrm{e}, \mathrm{h}) \mathcal{P}_{0} \approx 10^{3} \mathcal{P}_{c}$ (marked with short vertical dashes), where $\mathcal{P}_{c}=(2 \pi)^{2} \mathcal{E}_{0}$ is the Poincaré limit indicated with vertical dash-dotted lines in figures (a) and (b). 


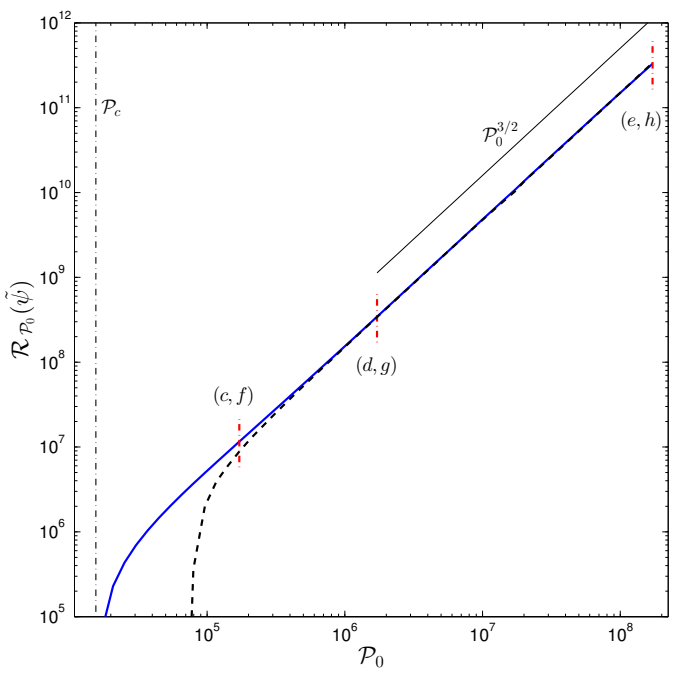

(a)

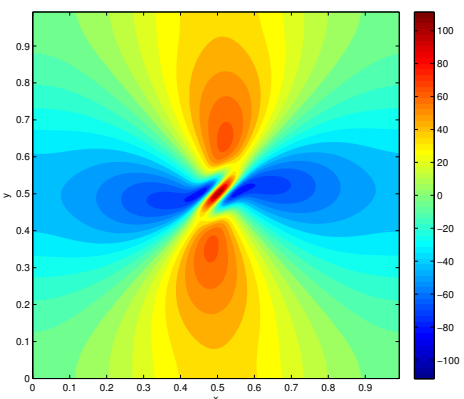

(c)

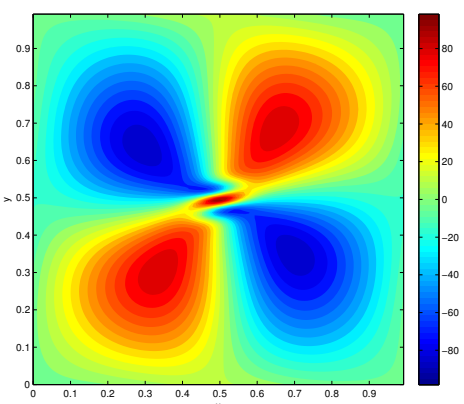

(f)

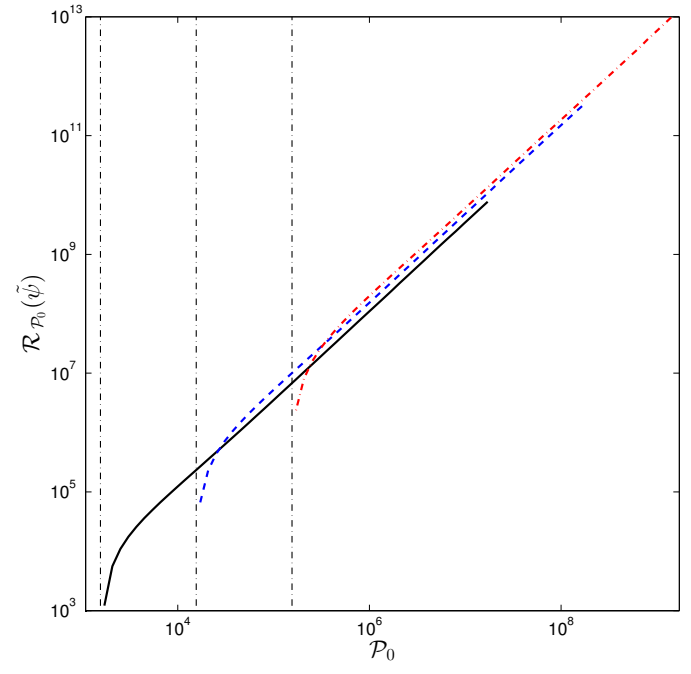

(b)

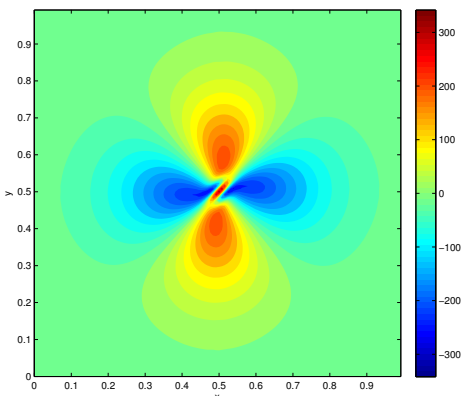

(d)

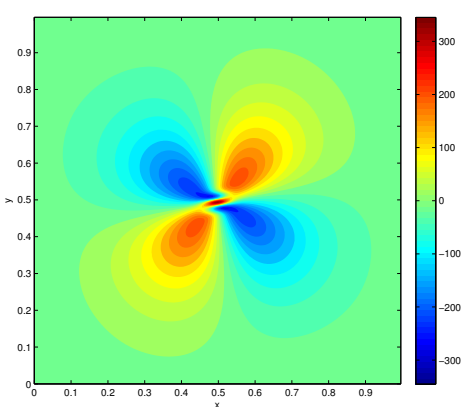

(g)

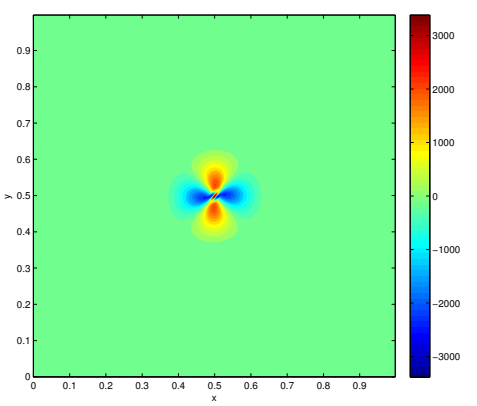

(e)

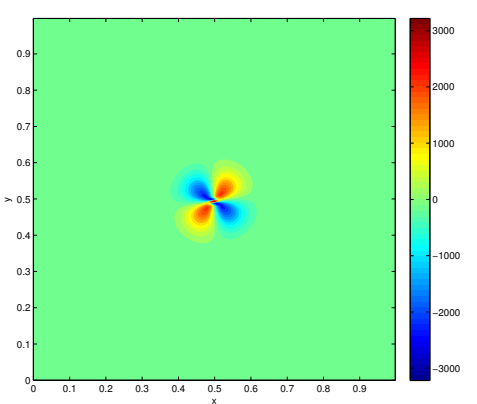

(h)

Figure 4: Dependence of the maximum palinstrophy rate of growth $\mathcal{R}_{\mathcal{P}_{0}}\left(\tilde{\psi}_{\mathcal{K}_{0}, \mathcal{P}_{0}}\right)$ on $\mathcal{P}_{0}$ for $(\mathrm{a}) \mathcal{K}_{0}=10$ and (b) $\mathcal{K}_{0}=10^{0}, 10^{1}$ and $10^{2}$. Figure (a) shows both solution branches, whereas figure (b) only the ones with larger values of $\mathcal{R}_{\mathcal{P}_{0}}$. Optimal vortex states corresponding to the two branches are shown in figures $(\mathrm{c}-\mathrm{e})$ and $(\mathrm{f}-\mathrm{h})$ for the following palinstrophy values: $(\mathrm{c}, \mathrm{f}) \mathcal{P}_{0}=10 \mathcal{P}_{c},(\mathrm{~d}, \mathrm{~g}) \mathcal{P}_{0}=10^{2} \mathcal{P}_{c}$ and $(\mathrm{e}, \mathrm{h}) \mathcal{P}_{0}=10^{4} \mathcal{P}_{c}$ (marked with short vertical dashes), where $\mathcal{P}_{c}=(2 \pi)^{4} \mathcal{K}_{0}$ is the Poincaré limit indicated with vertical dash-dotted lines in figures (a) and (b). 
This behavior is parallel to the behavior reported in sections 6.1 and 6.2 where power-law scaling was observed in the single-constraint problem and in the $\left(\mathcal{K}_{0}, \mathcal{P}_{0}\right)$-constrained problem, but not in the $\left(\mathcal{E}_{0}, \mathcal{P}_{0}\right)$-constrained problem. The exponents characterizing power laws (45) are significantly smaller than 2 predicted by estimate (13). The dependence of $\mathcal{P}_{\max }$ on $\mathcal{E}_{0}$ is cases (i) and (ii) is shown in figure $5(\mathrm{~b})$ ( $\mathcal{E}_{0}$ rather than $\mathcal{P}_{0}$ is chosen as the abscissa, since this is the "independent variable" in the nonlinear term in estimate (14), and case (iii) is not shown, because in this configuration $\mathcal{E}_{0}$ is fixed). The following two distinct power laws are observed in the two cases

$$
\begin{aligned}
\text { (i) } \mathcal{P}_{\max } & \sim \mathcal{E}_{0}^{1.17 \pm 0.02} \\
\text { (ii) } \mathcal{P}_{\max } & \sim \mathcal{E}_{0}^{1.98 \pm 0.07}
\end{aligned}
$$

implying that the maximizing vortex states obtained under the $\left(\mathcal{K}_{0}, \mathcal{P}_{0}\right)$-constraint lead to a finite-time palinstrophy evolution which also saturates the finite-time estimate (14). The significance of this finding will be discussed in more detail in the following section. In figure $5(\mathrm{c})$ we show the time evolution of the palinstrophy $\mathcal{P}(t)$ corresponding to case (ii) with $\mathcal{K}_{0}=10$ and $\mathcal{P}_{0}=10^{4} \mathcal{P}_{c}$, where $\mathcal{P}_{c}=(2 \pi)^{4} \mathcal{K}_{0}$ is the Poincaré limit, which is representative of the entire family. We note that the initially steep growth of the palinstrophy is followed by its eventual viscous decay. The time $T_{\max }:=\operatorname{argmax}_{t \geq 0} \mathcal{P}(t)$ when the maximum is attained depends on the initial palinstrophy $\mathcal{P}_{0}$ exhibiting a welldefined power-law, cf. figure 5(d),

$$
T_{\max } \sim \mathcal{P}_{0}^{-0.47 \pm 0.06}
$$

We remark that scaling with the same exponent was also observed in the evolution leading to the maximum finite-time growth of enstrophy in the 1D Burgers problem (Ayala \& Protas, 2011; Pelinovsky, 2012b). For a detailed discussion of the vortex dynamics mechanisms responsible for the evolution leading to power-law (46)(ii), we refer the reader to the companion paper by Ayala \& Protas (2013). The exponents characterizing all the power laws discussed in this section are collected in table 2.

\section{Discussion}

In this section we comment on some of the theoretical results introduced in section 2 in the light of the findings presented in section 6. Our main interest here is establishing the sharpness of estimates (10)-(12) for $d \mathcal{P} / d t$, and from figures $4(\mathrm{a}, \mathrm{b})$ and table 2 we see that solutions of optimization problem (19) lead to the growth of $\mathcal{R}_{\mathcal{P}_{0}}$ which saturates estimate (11) for sufficiently large $\mathcal{P}_{0}$, so that we conclude that this estimate is sharp. As regards upper bound (10), due to the presence of the negative quadratic term, this estimate does not have the form of a power-law allowing for an arbitrary growth of $\mathcal{R}_{\mathcal{P}_{0}}\left(\tilde{\psi}_{\mathcal{E}_{0}, \mathcal{P}_{0}}\right)$. This is indeed confirmed by the behavior of the solutions of optimization problem (18) shown in Figures $3(\mathrm{a}, \mathrm{b})$ where we see that on each branch the quantity $\mathcal{R}_{\mathcal{P}_{0}}\left(\tilde{\psi}_{\mathcal{E}_{0}, \mathcal{P}_{0}}\right)$ eventually decreases with $\mathcal{P}_{0}$. Hence, in this case a power-law cannot be 


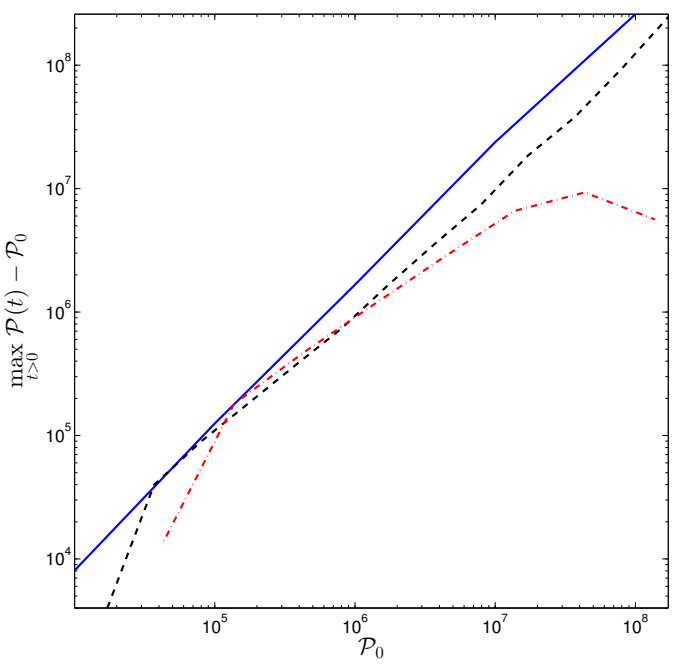

(a)

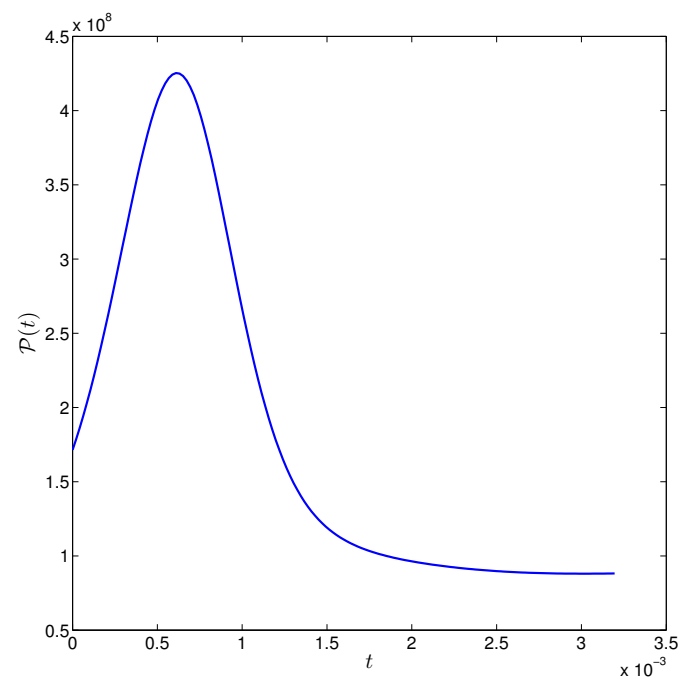

(c)

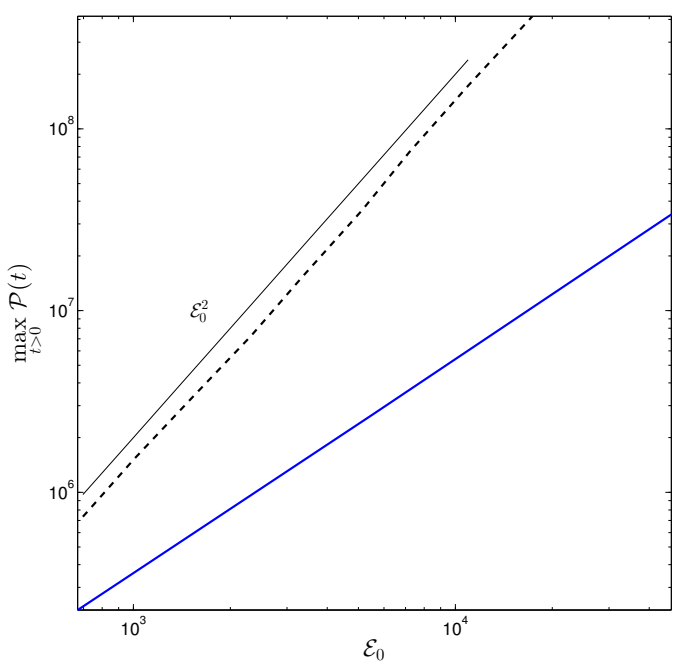

(b)

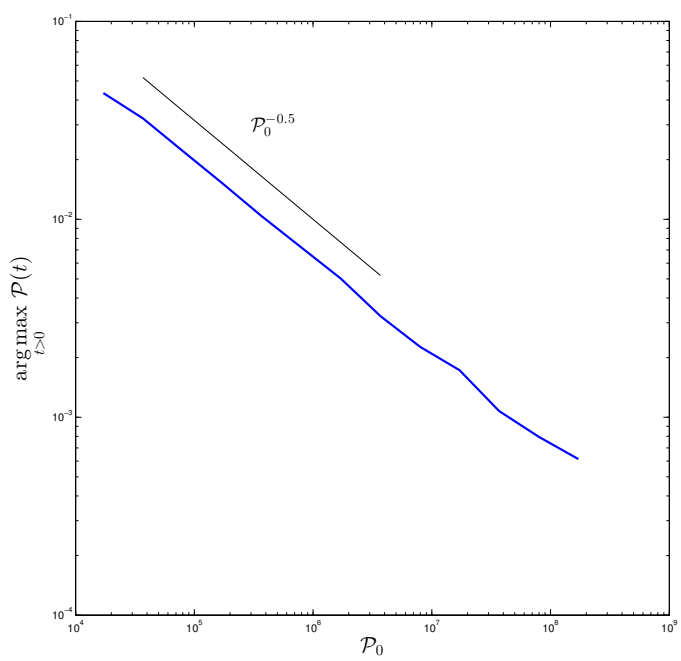

(d)

Figure 5: (a) $\delta \mathcal{P}$ as a function of initial palinstrophy $\mathcal{P}_{0}$ for (solid) case (i), (dashed) case (ii) and (dotted) case (iii); (b) $\mathcal{P}_{\max }$ as a function of initial enstrophy $\mathcal{E}_{0}$ for (solid) case (i) and (dashed) case (ii); (c) an individual time-history of $\mathcal{P}(t)$ in case (ii) corresponding to $\mathcal{K}_{0}=10$ and $\mathcal{P}_{0}=10^{4} \mathcal{P}_{c}$, where $\mathcal{P}_{c}=(2 \pi)^{4} \mathcal{K}_{0}$ is the Poincaré limit; (d) time $T_{\max }$ when the maximum palinstrophy is attained as a function of the initial palinstrophy $\mathcal{P}_{0}$ in case (ii); see text in section 6.3 for the definition of each case. 


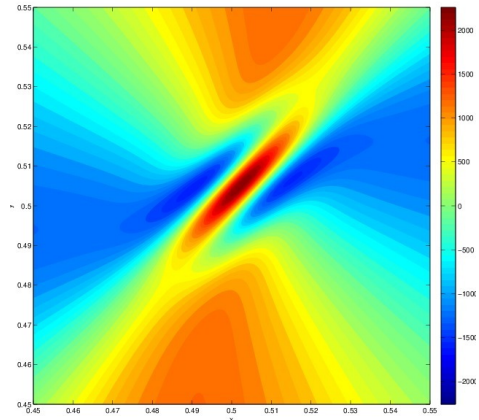

(a)

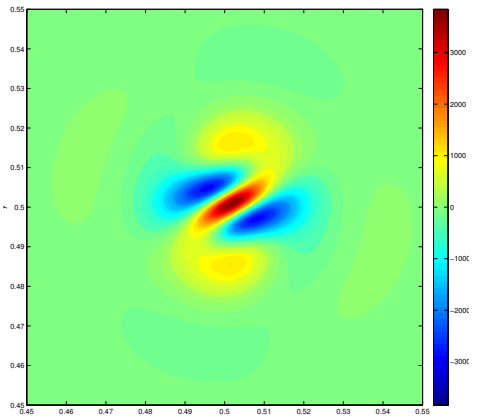

(b)

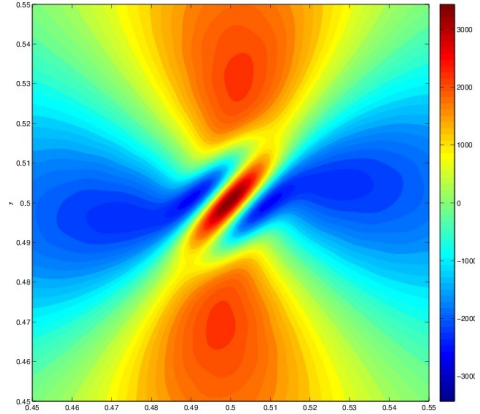

(c)

Figure 6: Magnifications of localized vortex structures present in the maximizing vorticity fields (a) $-\Delta \tilde{\psi}_{\mathcal{P}_{0}}$, (b) $-\Delta \tilde{\psi}_{\mathcal{E}_{0}, \mathcal{P}_{0}}$ and (c) $-\Delta \tilde{\psi}_{\mathcal{K}_{0}, \mathcal{P}_{0}}$ with $\mathcal{P}_{0} \approx 10^{8}$ in all three cases, $\mathcal{E}_{0}=10^{3}$ in (b) and $\mathcal{K}_{0}=10 \mathrm{in} \mathrm{(c).}$

in fact defined. Since the qualitative features of estimate (10) are reproduced by the actual dependence of $\mathcal{R}_{\mathcal{P}_{0}}\left(\tilde{\psi}_{\mathcal{E}_{0}, \mathcal{P}_{0}}\right)$ on $\mathcal{P}_{0}$ observed in figures $3(\mathrm{a}, \mathrm{b})$, we can conclude that this estimate predicts the correct behavior, although in the absence of a power-law, it is hard to quantify this statement in terms of exponents. It is interesting that optimization problems (18) and (19) which have a rather similar structure lead to quite different global behavior of the maximizing solutions. The reason for this is that, as the palinstrophy $\mathcal{P}_{0}$ is increased, in the $\left(\mathcal{E}_{0}, \mathcal{P}_{0}\right)$-constrained family of optimizers the energy $\mathcal{K}\left(\tilde{\psi}_{\mathcal{E}_{0}, \mathcal{P}_{0}}\right)$ can not increase arbitrarily, as it is upper-bounded by $\mathcal{E}_{0}$ via Poincaré's inequality. On the other hand, in the $\left(\mathcal{K}_{0}, \mathcal{P}_{0}\right)$-constrained family of optimizers the constraint on $\mathcal{K}_{0}$ does not limit the growth of the enstrophy $\mathcal{E}\left(\tilde{\psi}_{\mathcal{K}_{0}, \mathcal{P}_{0}}\right)$ of the maximizing solutions.

In regard to the finite-time estimates, the fact that upper bound (14) is saturated by the evolution corresponding to the $\left(\mathcal{K}_{0}, \mathcal{P}_{0}\right)$-constrained maximizers is intriguing. We recall that the maximizers found to saturate the instantaneous estimates in $1 \mathrm{D}$ subject to one constraint only (on $\mathcal{E}$ ) did not lead to evolution saturating the corresponding finitetime estimates ( $\mathrm{Lu} \&$ Doering, 2008; Ayala \& Protas, 2011). The role of the number of the constraints imposed on the solutions in this type of optimization problems deserves further study.

Moving on to estimate (12) and solutions of the single-constraint optimization problem $(20)$, we observe in figure $2(\mathrm{~b})$ that while $\mathcal{R}_{\mathcal{P}_{0}}\left(\tilde{\psi}_{\mathcal{P}_{0}}\right)$ exhibits a very clean power-law dependence on $\mathcal{P}_{0}$, the associated exponent is in fact significantly less than 2 predicted by estimate (12), cf. table 2 . This was in fact to be expected, since upper bound (12) was obtained with the use of Poincaré's inequality which is saturated only by the eigenfunctions of the Laplacian operator and, as is evident from figure $2(\mathrm{e}, \mathrm{h})$, the maximizing solutions $\tilde{\psi}_{\mathcal{P}_{0}}$ for large $\mathcal{P}_{0}$ are quite different from such eigenfunctions. We add that analogous instantaneous estimates in 1D and in 3D were in fact found to be sharp by Lu \& Doering (2008), cf. table 1. 
We now comment on the structure of the maximizing vorticity fields. First, we observe that in all three optimization problems there are two branches of locally maximizing solutions, cf. figures 2, 3 and 4, and they are obtained via continuation from the limiting, for small $\mathcal{P}_{0}$, solutions which were characterized analytically in section 5 . In that section we also observed that in the limit of small $\mathcal{P}_{0}$ the cubic term in $\mathcal{R}_{\mathcal{P}_{0}}(\psi)$ vanishes, cf. (39), so that the maximizing fields sustain no stretching of the vorticity gradients. Interestingly, since these limiting maximizers satisfy equation (37a) (which is a special case of $\Delta \psi=F(\psi)$ with a particular $F: \mathbb{R} \rightarrow \mathbb{R}$ ), they are also steady solutions of the 2D Euler equations (Majda \& Bertozzi, 2002). When used as the initial data in a 2D time-dependent Navier-Stokes problem (3), they give rise to the Taylor-Green vortex flow characterized by a purely exponential decay without any nonlinear interactions. We add that 3D generalizations of this flow lead to nontrivial time evolution and have been investigated in the context of the finite-time blow-up problem (Brachet et al., 1983; Brachet, 1991). The relationship between the symmetry of vortex configurations and the amplification of the vorticity via stretching was studied by Pelz (2001).

As the palinstrophy $\mathcal{P}_{0}$ increases, the maximizers in all three optimization problems become localized multipolar vortex structures shown in figures $6(\mathrm{a}-\mathrm{c})$ and featuring a central elongated filament stretched by four satellite vortices: two stronger ones which are closer to the central filament and have the opposite sign, and two weaker ones which are further away and have the same sign as the central filament. In the absence of this central filament, the four satellite vortices would resemble the axial vorticity distribution in the meridional plane intersecting two parallel vortex rings, which was in fact the optimal vortex state found by $\mathrm{Lu} \&$ Doering (2008, see Figure 4.6a) to saturate the 3D instantaneous estimate (1). This observation offers some analogy to the $3 \mathrm{D}$ problem with the presence of the central vortex filament reflecting the difference in the physical quantities maximized in the two problems: vorticity (enstrophy) in 3D versus vorticity gradients (palinstrophy) in $2 \mathrm{D}$. In the limit of large $\mathcal{P}_{0}$, the vortex states corresponding to the two branches appear very similar, except for the rotation by a $45 \mathrm{deg}$ angle. The difference between the cases with one and two constraints is that in the former case the satellite vortices tend to be less localized (which is a consequence of the fact that in that case $\mathcal{K}_{0}$ and $\mathcal{E}_{0}$ can change freely). With increasing palinstrophy $\mathcal{P}_{0}$, the optimal vortex structures in the single-constraint and $\left(\mathcal{K}_{0}, \mathcal{P}_{0}\right)$-constrained cases shrink in a shape-preserving manner with the characteristic dimension $\Lambda$ of the vortex structure vanishing while its magnitude $\omega_{\max }$ grows, so that the vorticity fields can be empirically approximated by the asymptotic formula $\omega_{\mathcal{P}_{0}}(\mathbf{x}) \sim \omega_{\max } \Pi(\mathbf{x} / \Lambda)$ for some distribution $\Pi$ independent of $\mathcal{P}_{0}$. Figure 7 shows the dependence of the quantities $\Lambda=$ $2 \pi \sqrt{\mathcal{K}\left(\tilde{\psi}_{\mathcal{K}_{0}, \mathcal{P}_{0}}\right) / \mathcal{E}\left(\tilde{\psi}_{\mathcal{K}_{0}, \mathcal{P}_{0}}\right)}$, cf. (Doering \& Gibbon, 1995), and $\omega_{\max }=\left\|\Delta \tilde{\psi}_{\mathcal{K}_{0}, \mathcal{P}_{0}}\right\|_{L_{\infty}(\Omega)}$ computed for the upper branch of $\left(\mathcal{K}_{0}, \mathcal{P}_{0}\right)$-constrained maximizers on $\mathcal{P}_{0}$ with $\mathcal{K}_{0}=1$, cf. figure 4 . This data reveals clear power laws $\Lambda \sim \mathcal{P}_{0}^{-1 / 4}$ and $\omega_{\max } \sim \mathcal{P}_{0}^{1 / 2}$ holding for sufficiently large $\mathcal{P}_{0}$ which confirms the scale-independent structure of the maximizing vortex states. Obtaining an analytical characterization of the maximizers $\tilde{\psi}_{\mathcal{P}_{0}}$ and $\tilde{\psi}_{\mathcal{K}_{0}, \mathcal{P}_{0}}$ in the limit $\mathcal{P}_{0} \rightarrow \infty$ is an interesting open research problem in mathematical analysis. 


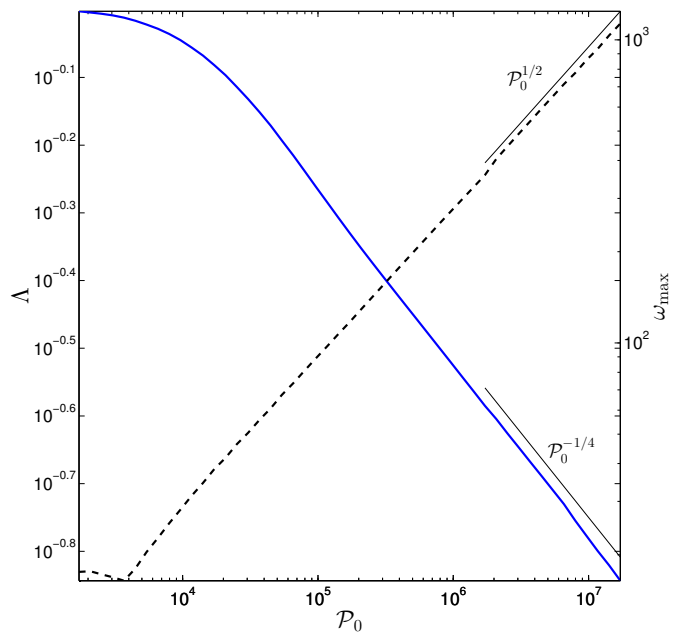

Figure 7: Dependence of (solid) the characteristic length scale $\Lambda=$ $2 \pi \sqrt{\mathcal{K}\left(\tilde{\psi}_{\mathcal{K}_{0}, \mathcal{P}_{0}}\right) / \mathcal{E}\left(\tilde{\psi}_{\mathcal{K}_{0}, \mathcal{P}_{0}}\right)}$ and (dashed) the vorticity magnitude $\omega_{\max }=\left\|\Delta \tilde{\psi}_{\mathcal{K}_{0}, \mathcal{P}_{0}}\right\|_{L_{\infty}(\Omega)}$ on $\mathcal{P}_{0}$ with $\mathcal{K}=1$ for the family of maximizers obtained subject to the $\left(\mathcal{K}_{0}, \mathcal{P}_{0}\right)$ constraint (cf. figure 4).

Finally, we observe that both families of the maximizing solutions shown in figures 2-4 exhibit an interesting pattern. While for decreasing $\mathcal{P}_{0}$ the maximizing solutions approach the Laplacian eigenfunctions with either aligned or staggered arrangement of the vortex cells, cf. (40)-(42) and figure 1 , for increasing $\mathcal{P}_{0}$ the dominating vortex structure is shifted to the stagnation point of the maximizing fields corresponding to small $\mathcal{P}_{0}$. Furthermore, the dominating vortex structure in the limit of large $\mathcal{P}_{0}$ is aligned with the direction of the maximum stretching characterizing the maximizer in the low $\mathcal{P}_{0}$ limit, i.e., vertically/horizontally for the aligned arrangement and inclined at the angle of $45 \mathrm{deg}$ for the staggered arrangement. This pattern is schematically illustrated in figure 8 .

\section{Conclusions and Outlook}

In this investigation we addressed a problem which is a part of a broader research program concerning characterization of the maximum growth of certain quadratic quantities in the hydrodynamic systems in different spatial dimensions (cf. table 1). Here we focused on the upper bounds for the instantaneous rate of growth of palinstrophy $d \mathcal{P} / d t$ and demonstrated that certain available estimates are in fact sharp with respect to variations of the palinstrophy and are saturated by families of vorticity fields with nontrivial structure. Estimate (11) was found to be realizable, even though there exist other estimates, cf. (15), with the RHS involving a lower power of $\mathcal{P}$, which is a consequence 


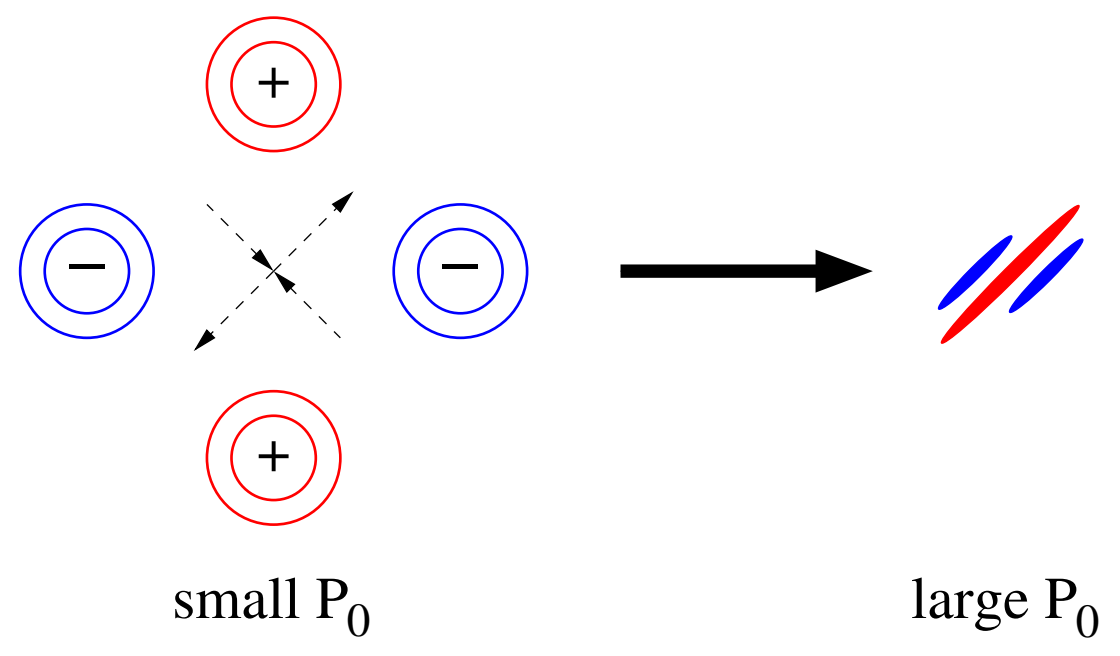

Figure 8: Schematic illustration of the change of the structure of the local maximizers in the staggered arrangement of the vortex cells as $\mathcal{P}_{0}$ increases (dashed lines represent the principal directions of stretching and compression).

\begin{tabular}{c|c|c} 
Estimate & Constraints & Computed Power-Law \\
\hline$\frac{d \mathcal{P}}{d t} \leq \nu \frac{\mathcal{P}^{2}}{\mathcal{E}}+\frac{C}{\nu} \mathcal{E} \mathcal{P}[\mathrm{cf.}(10)]$ & $\mathcal{E}_{0}, \mathcal{P}_{0}$ & Not applicable \\
\hline$\frac{d \mathcal{P}}{d t} \leq \frac{C}{\nu} \mathcal{K}^{\frac{1}{2}} \mathcal{P}^{\frac{3}{2}}[\mathrm{cf.}(11)]$ & $\mathcal{K}_{0}, \mathcal{P}_{0}$ & $\frac{d \mathcal{P}}{d t} \sim \mathcal{P}_{0}^{1.49 \pm 0.02}$ \\
\hline$\frac{d \mathcal{P}}{d t} \leq \frac{C}{\nu} \mathcal{P}^{2}[\mathrm{cf.}(12)]$ & $\mathcal{P}_{0}$ & $\frac{d \mathcal{P}}{d t} \sim \mathcal{P}_{0}^{1.57 \pm 0.05}$ \\
\hline $\max _{t>0} \mathcal{P}(t) \leq \mathcal{P}_{0}+\frac{C}{\nu^{2}} \mathcal{E}_{0}^{2}[\mathrm{cf.}(13)]$ & $\mathcal{P}_{0}$ & $\max _{t>0} \mathcal{P}(t) \sim \mathcal{E}_{0}^{1.17 \pm 0.02}$ \\
\hline $\max _{t>0} \mathcal{P}(t) \leq\left(\mathcal{P}_{0}^{1 / 2}+\frac{C_{2}}{4 \nu^{2}} \mathcal{K}_{0}^{1 / 2} \mathcal{E}_{0}\right)^{2}[\mathrm{cf.}(14)]$ & $\mathcal{K}_{0}, \mathcal{P}_{0}$ & $\max _{t>0} \mathcal{P}(t) \sim \mathcal{E}_{0}^{1.98 \pm 0.07}$ \\
\hline $\mathrm{N} / \mathrm{A}$ & $\mathcal{K}_{0}, \mathcal{P}_{0}$ & $\operatorname{argmax}_{t \geq 0} \mathcal{P}(t) \sim \mathcal{P}_{0}^{-0.47 \pm 0.06}$
\end{tabular}

Table 2: Comparison of the analytical estimates from section 2 and the power-laws discovered by solving the maximization problem in sections 6.1 and 6.2 , and the initialvalue problems in section 6.3 . 
of the choice of the quantities constraining the upper bound expression $\left(\mathcal{K}_{0}^{1 / 2}\right.$ versus $\left.\left\|\omega_{0}\right\|_{L_{\infty}(\Omega)}\right)$. The optimal vortex states with prescribed energy $\mathcal{K}_{0}$ and palinstrophy $\mathcal{P}_{0}$ were also found to lead to a time-evolution saturating the finite-time estimate, which is an interesting result highlighting the role the number of constraints may play in this type of problems. Sharpness of finite-time estimates can also be assessed by solving optimization problems defined over finite windows of time, as done by Ayala \& Protas (2011) for the 1D Burgers equation. It is interesting to see whether such an approach could lead to an improved prefactor in power law (46)(ii). We intend to address this question in the future. As regards the present study, another interesting question is how our findings would change if the optimization problems were formulated in an unbounded, rather than periodic, domain. Sharpness of estimates (15)-(16), which have a rather different structure than bounds (11) and (14), with respect to variations of the palinstrophy $\mathcal{P}_{0}$ is also an interesting open question, however, due to the presence of the nondifferentiable factor $\|\omega\|_{L_{\infty}}$ on the RHS, the corresponding variational optimization problems will be nonsmooth and require highly specialized methods for their numerical solution. These estimates appear sharper than (11) and (14) with respect to variations of the viscosity $\nu$, and it would also be interesting to examine their realizability in such terms. A separate set of questions concerns flows on domains with rigid boundaries and some results relevant to the blow-up problem in such setting were recently reported by Gibbon \& Titi (2013).

Concerning the research program presented in Introduction, quantifying the maximum finite-time growth of enstrophy in the 3D Navier-Stokes system remains of course the ultimate goal, one which we hope is within reach in the foreseeable future given the currently available computational tools and resources. An interesting intermediate step is to consider similar questions for the $2 \mathrm{D}$ surface quasi-geostrophic (SQG) equation

$$
\frac{\partial \theta}{\partial t}+(\mathbf{v} \cdot \nabla) \theta=-\nu(-\Delta)^{\alpha} \theta
$$

where $\mathbf{v}=\nabla^{\perp}(-\Delta)^{-1 / 2} \theta$ and $0 \leq \alpha \leq 1$. As is suspected (Kiselev, 2010; Scott, 2011), solutions of (48) may exhibit finite-time blow-up in the supercritical case $\alpha<1 / 2$. Therefore, aside from its own intrinsic interest, this problem represents a useful testbed for development and validation of methods to track singular solutions in the 2D setting which is more computationally manageable than the full 3D Navier-Stokes problem.

We wish to emphasize that the research methodology developed here, relying on a systematic characterization of the extremal behavior, appears applicable to other related open problems in the field of theoretical fluid dynamics. An example of such a problem is obtaining sharp bounds on the Nusselt number in the Raleigh-Bénard convection (Hassanzadeh et al., 2013). There are also similar problems related to mixing.

\section{Acknowledgements}

The authors are indebted to Charles Doering and Evelyn Lunasin for many enlightening discussions concerning the research problems studied in this work and, in particular, for 
providing estimates (10) and (13). They are also thankful to an anonymous referee for many insightful comments concerning, in particular, estimate (17) and its relationship to other bounds considered in this study. The authors are grateful to Nicholas Kevlahan for making his parallel Navier-Stokes solver available, which was used to obtain the results reported in section 6.3. This research was funded through an Early Researcher Award (ERA) and an NSERC Discovery Grant. The computational time was made available by SHARCNET.

\section{A Derivation of Estimates (11) and (14)}

A key element necessary to derive the upper bound in (11) is the following estimate for the $L_{\infty}$ norm of doubly-periodic functions $\mathbf{u}: \Omega \rightarrow \mathbb{R}^{2}$

$$
\|\mathbf{u}\|_{\infty} \leq C\|\mathbf{u}\|_{2}^{1 / 2}\|\Delta \mathbf{u}\|_{2}^{1 / 2}
$$

where $C>0$, which follows from Sobolev Interpolation Theorem (Adams \& Fournier, 2005). A calculation showing that for $\Omega=[0,1]^{2}, C=1 / \sqrt{\pi}$ can be found in Ayala (2014).

We notice that the estimate depends only on the $L_{2}$ norms of the function and some of its second derivatives. To obtain estimate (11) from section 2, we write the rate of growth of palinstrophy as, cf. (9),

$$
\frac{d \mathcal{P}}{d t}=-\nu \int_{\Omega}|\Delta \omega|^{2} d \Omega+\int_{\Omega} \mathbf{u} \cdot \nabla \omega \Delta \omega d \Omega .
$$

The second term on the RHS in (50) can be upper-bounded as

$$
\begin{aligned}
\left|\int_{\Omega} \mathbf{u} \cdot \nabla \omega \Delta \omega d \Omega\right| & \leq\|\mathbf{u} \cdot \nabla \omega\|_{2}\|\Delta \omega\|_{2} \\
& \leq\|\mathbf{u}\|_{\infty}\|\nabla \omega\|_{2}\|\Delta \omega\|_{2} \\
& \leq C\|\mathbf{u}\|_{2}^{1 / 2}\|\Delta \mathbf{u}\|_{2}^{3 / 2}\|\Delta \omega\|_{2}
\end{aligned}
$$

where inequality (49) has been used together with the Cauchy-Schwarz inequality and the fact that $\|\nabla \omega\|_{2}=\|\Delta \mathbf{u}\|_{2}$. The application of Young's inequality

$$
a b \leq \frac{\beta^{p} a^{p}}{p}+\frac{b^{q}}{q \beta^{q}}
$$

to $(50)$ with $p=q=2$ and $\beta^{2}=(2 \nu)^{-1}$ yields

$$
\begin{aligned}
\frac{d \mathcal{P}}{d t} & \leq-\nu\|\Delta \omega\|_{2}^{2}+\frac{C^{2}}{4 \nu}\|\mathbf{u}\|_{2}\|\Delta \mathbf{u}\|_{2}^{3}+\nu\|\Delta \omega\|_{2}^{2} \\
& \leq \frac{C^{2}}{4 \nu}\|\mathbf{u}\|_{2}\|\Delta \mathbf{u}\|_{2}^{3}
\end{aligned}
$$


Finally, inequality (51) can be rewritten in terms of energy and palinstrophy as, cf. (11),

$$
\frac{d \mathcal{P}}{d t} \leq \frac{C^{2}}{\nu} \mathcal{K}^{1 / 2} \mathcal{P}^{3 / 2}
$$

It follows from Navier-Stokes system (3) that $d \mathcal{K} / d t=-2 \nu \mathcal{E}$ and $d \mathcal{E} / d t=-2 \nu \mathcal{P}$. Therefore, $\mathcal{K}(t) \leq \mathcal{K}(0)=\mathcal{K}_{0}$ and $\mathcal{E}(t) \leq \mathcal{E}(0)=\mathcal{E}_{0}$ for all $t>0$. Estimate (52) can be transformed as

$$
\begin{aligned}
\frac{d \mathcal{P}}{d t} & \leq \frac{C^{2}}{\nu} \mathcal{K}_{0}^{1 / 2} \mathcal{P}^{3 / 2} \quad \Longrightarrow \\
\mathcal{P}^{-1 / 2} \frac{d \mathcal{P}}{d t} & \leq \frac{C^{2}}{\nu} \mathcal{K}_{0}^{1 / 2} \mathcal{P} \quad \Longrightarrow \\
\frac{d}{d t}\left(2 \mathcal{P}^{1 / 2}\right) & \leq \frac{C^{2}}{\nu} \mathcal{K}_{0}^{1 / 2} \mathcal{P} .
\end{aligned}
$$

Integrating the last inequality over time and using the fact that

$$
\int_{0}^{t} \mathcal{P}(s) d s=\frac{\mathcal{E}(0)-\mathcal{E}(t)}{2 \nu} \leq \frac{\mathcal{E}_{0}}{2 \nu},
$$

it is possible to obtain

$$
\mathcal{P}(t) \leq\left[\mathcal{P}_{0}^{1 / 2}+\left(\frac{C}{2 \nu}\right)^{2} \mathcal{K}_{0}^{1 / 2} \mathcal{E}_{0}\right]^{2} .
$$

This upper bound is valid for all $t>0$ and the right-hand side depends only on the initial values of energy, enstrophy and palinstrophy. Estimate (14) is then obtained by taking the maximum over time on the LHS in (53).

\section{References}

Adams, R. A. \& Fournier, J. F. 2005 Sobolev Spaces. Elsevier.

AyAla, D. 2014 Extreme vortex states and singularity formation. PhD thesis, McMaster University, in preparation.

Ayala, D. \& Protas, B. 2011 On maximum enstrophy growth in a hydrodynamic system. Physica D 240, 1553-1563.

Ayala, D. \& Protas, B. 2013 Vortices, maximum growth and the problem of finitetime singularity formation. Fluid Dynamics Research (accepted for publication).

Brachet, M. E. 1991 Direct simulation of three-dimensional turbulence in the TaylorGreen vortex. Fluid Dynamics Research 8, 1-8. 
Brachet, M. E., Meiron, D. I., Orszag, S. A., Nickel, B. G., Morf, R. H. \& FRISCH, U. 1983 Small-scale structure of the Taylor-Green vortex. Journal of Fluid Mechanics 130, 411-452.

Bustamante, M. D. \& Brachet, M. 2012 Interplay between the Beale-Kato-Majda theorem and the analyticity-strip method to investigate numerically the incompressible Euler singularity problem. Phys. Rev. E 86, 066302.

Dascaliuc, R., Foias, C. \& Jolly, M. S. 2010 Estimates on enstrophy, palinstrophy, and invariant measures for 2D turbulence. Journal of Differential Equations 248, 792819.

Doering, C. R. 2009 The 3D Navier-Stokes problem. Annual Review of Fluid Mechanics pp. 109-128.

Doering, C. R. \& Gibbon, J. D. 1995 Applied Analysis of the Navier-Stokes Equations. Cambridge University Press.

Doering, C. R. \& Lunasin, E. 2011 Limits on palinstrophy growth for solutions of the two-dimensional Navier-Stokes equations. Personal communication.

Edwards, W. S., Tuckerman, L. S., Friesner, R. A. \& Sorensen, D. C. 1994 Krylov method for the incompressible Navier-Stokes equation. Journal of Computational Physics 110, 82-102.

Farazmand, M., Kevlahan, N. K. R. \& Protas, B. 2011 Controlling the dual cascade of two-dimensional turbulence. Journal of Fluid Mechanics 668, 202-222.

Fefferman, C. L. 2000 Existence and smoothness of the Navier-Stokes equation. available at http://www.claymath.org/millennium/Navier-Stokes_Equations/ navierstokes.pdf, Clay Millennium Prize Problem Description.

Foias, C. \& Temam, R. 1989 Gevrey class regularity for the solutions of the NavierStokes equations. Journal of Functional Analysis 87, 359-369.

Gibbon, J. D., Bustamante, M. \& Kerr, R. M. 2008 The three-dimensional Euler equations: singular or non-singular? Nonlinearity 21, 123-129.

Gibbon, J. D. \& Titi, E. S. 2013 The 3D incompressible Euler equations with a passive scalar: a road to blow-up? ArXiv:1211.3811.

Grafke, T., Homann, H., Dreher, J. \& Grauer, R. 2008 Numerical simulations of possible finite-time singularities in the incompressible Euler equations: comparison of numerical methods. Physica D 237, 1932-1936.

Gunzburger, M. D. 2003 Perspectives in Flow Control and Optimization. SIAM. 
Hassanzadeh, P., Chini, G. P. \& Doering, C. R. 2013 Wall to wall optimal transport. ArXiv:1309.5542.

Hou, T. Y. 2009 Blow-up or no blow-up? a unified computational and analytic approach to 3D incompressible Euler and Navier-Stokes equations. Acta Numerica pp. $277-346$.

KERR, R. M. 1993 Evidence for a singularity of the three-dimensional, incompressible Euler equations. Phys. Fluids A 5, 1725-1746.

Kiselev, A. 2010 Regularity and blow up for active scalars. Math. Model. Nat. Phenom. $5,225-255$.

Kreiss, H. \& Lorenz, J. 2004 Initial-Boundary Value Problems and the Navier-Stokes Equations, Classics in Applied Mathematics, vol. 47. SIAM.

LadyZhenskayA, O. A. 1969 The Mathematical Theory of Viscous Incompressible Flow. Gordon and Breach.

LU, L. 2006 Bounds on the enstrophy growth rate for solutions of the 3D Navier-Stokes equations. PhD thesis, University of Michigan.

Lu, L. \& Doering, C. R. 2008 Limits on enstrophy growth for solutions of the threedimensional Navier-Stokes equations. Indiana University Mathematics Journal 57, $2693-2727$.

Luenberger, D. 1969 Optimization by Vector Space Methods. John Wiley and Sons.

Majda, A. J. \& Bertozzi, A. L. 2002 Vorticity and Incompressible Flow. Cambridge University Press.

Matsumoto, T., Bec, J. \& Frisch, U. 2008 Complex-space singularities of 2D Euler flow in lagrangian coordinates. Physica D 237, 1951-1955.

Ohkitani, K. 2008 A miscellany of basic issues on incompressible fluid equations. Nonlinearity 21, 255-271.

Ohkitani, K. \& Constantin, P. 2008 Numerical study of the eulerian-lagrangian analysis of the Navier-Stokes turbulence. Phys. Fluids 20, 1-11.

Orlandi, P., Pirozzoli, S. \& Carnevale, G. F. 2012 Vortex events in Euler and Navier-Stokes simulations with smooth initial conditions. Journal of Fluid Mechanics 690, 288-320.

Pelinovsky, D. $2012 a$ Enstrophy growth in the viscous Burgers equation. Dynamics of Partial Differential Equations 9, 305-340. 
Pelinovsky, D. $2012 b$ Sharp bounds on enstrophy growth in the viscous Burgers equation. Proceedings of Royal Society A 468, 3636-3648.

Pelz, R. B. 2001 Symmetry and the hydrodynamic blow-up problem. Journal of Fluid Mechanics 444, 299-320.

Pouquet, A., Lesieur, M., André, J. C. \& Basdevant, C. 1975 Evolution of high Reynolds number two-dimensional turbulence. Journal of Fluid Mechanics $\mathbf{7 2}$, 305-319.

Protas, B., Babiano, A. \& Kevlahan, N. K. R. 1999 On geometrical alignment properties of two-dimensional forced turbulence. Physica D 128, 169-179.

Protas, B., Bewley, T. \& Hagen, G. 2004 A comprehensive framework for the regularization of adjoint analysis in multiscale PDE systems. Journal of Computational Physics 195, 49-89.

Rabin, S. M. E., Caulfield, C. P. \& Kerswell, R. R. 2012 Variational identification of minimal seeds to trigger transition in plane Couette flow. Journal of Fluid Mechanics 712, 244-272.

RuszCZyŃski, A. 2006 Nonlinear Optimization. Princeton University Press.

Scott, R. K. 2011 A scenario for finite-time singularity in the quasigeostrophic model. Journal of Fluid Mechanics 687, 492-502.

SERrin, J. 1963 The initial value problem for the Navier-Stokes equations. In Nonlinear Problems (ed. Rudolph E. Langer), pp. 69-98. Mathematics Research Center, United States Army, The University of Wisconsin Press.

Siegel, M. \& Caflisch, R. E. 2009 Calculation of complex singular solutions to the 3D incompressible Euler equations. Physica D 238, 2368-2379.

Sulem, C., Sulem, P. L. \& Frisch, H. 1983 Tracing complex singularities with spectral methods. Journal of Computational Physics 50, 138-161.

Tran, Ch. V. \& Dritschel, D. G. 2006 Vanishing enstrophy dissipation in twodimensional Navier-Stokes turbulence in the inviscid limit. Journal of Fluid Mechanics 559, 107-116. 